\title{
Fluctuating vector mesons in analytically continued functional RG flow equations
}

\author{
Christopher Jung and Lorenz von Smekal \\ Institut für Theoretische Physik, Justus-Liebig-Universität, 35392 Giessen, Germany
}

(Received 1 October 2019; published 11 December 2019)

\begin{abstract}
In this work we study contributions due to vector and axial-vector meson fluctuations to their in-medium spectral functions in an effective low-energy theory inspired by the gauged linear sigma model. In particular, we show how to describe these fluctuations in the effective theory by massive (axial-)vector fields in agreement with the known structure of analogous single-particle or resonance contributions to the corresponding conserved currents. The vector and axial-vector meson spectral functions are then computed by numerically solving the analytically continued functional renormalization group flow equations for their retarded two-point functions at finite temperature and density in the effective theory. We identify the new contributions that arise due to the (axial-)vector meson fluctuations, and assess their influence on possible signatures of a QCD critical end point and the restoration of chiral symmetry in thermal dilepton spectra.
\end{abstract}

DOI: 10.1103/PhysRevD.100.116009

\section{INTRODUCTION}

Understanding the phases of strong-interaction matter and the transitions between them is still one of the main goals in theoretical and experimental heavy-ion physics [1-4]. In order to relate the experimental observations from heavy-ion collision experiments at various beam energies to the phase diagram of quantum chromodynamics (QCD) photons and dileptons are of particular importance to obtain information, e.g., on the temperature and the lifetime of the fireball [5-7]. They arise from various processes and are emitted in all stages of the collisions. Because they escape the fireball almost unaffected, these electromagnetic probes carry the spectral information of the matter in the interior of the collision zone during the evolution of the fireball. Therefore, the spectral properties of strong-interaction matter and the features of the QCD phase diagram, such as the in-medium modifications of hadrons reflecting chiral symmetry restoration and the transition to the quark-gluon plasma, are encoded in the measured photon and dilepton spectra [8].

The thermal rates are determined by the in-medium electromagnetic spectral function and can therefore be used to probe the QCD phase diagram [9]. Especially in the resonance region, for invariant masses of dilepton pairs below $1 \mathrm{GeV}$, the electromagnetic spectral function is dominated by vector mesons, in particular the $\rho$ meson

Published by the American Physical Society under the terms of the Creative Commons Attribution 4.0 International license. Further distribution of this work must maintain attribution to the author(s) and the published article's title, journal citation, and DOI. Funded by SCOAP . which gave rise to the famous vector-meson dominance (VMD) model [10]. Chiral symmetry on the other hand requires one to study the $\rho$ alongside its chiral partner, the $a_{1}$ meson. From the in-medium modifications to the spectral functions of vector and axial-vector mesons one then hopes to deduce the nature of chiral symmetry restoration and to find evidence of an associated critical end point in the phase diagram of QCD [6,11-13].

In this paper we compute the in-medium spectral functions of vector and axial-vector mesons in an extended linear sigma model with quarks as a chiral low-energy effective theory for two-flavor QCD. As in a previous study [14] we employ the functional renormalization group (FRG) as the nonperturbative computational framework to include fluctuations beyond mean-field approximations. The FRG has proven to be a powerful tool in diverse areas of physics, see [15-19] for reviews. One particular bonus that it shares with other functional methods here is its applicability, at least in principle, to the entire QCD phase diagram including the region of finite baryon density $[20,21]$ where lattice QCD suffers from the fermion sign problem. Mainly for technical reasons, applications to full QCD $[22,23]$ are nowadays often used to motivate effective low-energy models with the potential to constrain the input parameters at their upper limit of applicability, their ultraviolet (UV) scale. From this input, these can then be used to describe chiral symmetry restoration $[24,25]$ or model the deconfinement transition [26,27] with two or three quark flavors [28-30], and to describe vector mesons [31,32].

For real-time quantities such as spectral functions or transport coefficients one faces the additional problem, common to all Euclidean approaches to quantum field theory, of the analytic continuation from Euclidean back to 
Minkowski spacetime. Unlike lattice QCD, where numerical reconstruction methods are usually required, the FRG flow equations for correlation functions can be analytically continued before they are solved [33-35]. For a comparison of spectral functions from such analytically continued functional renormalization group (aFRG) flows to those from various reconstruction methods, see [36]. The power of the aFRG flow equations has by now been demonstrated in various model studies where mesonic and fermionic spectral functions have been computed [37-41], recently also self-consistently [42]. In order to assess truncation effects, one can furthermore compare with classical-statistical lattice simulations of spectral functions [43,44] which are able to capture exactly their universal critical behavior in the vicinity of continuous phase transitions.

Extending the previous study of aFRG flows for vector and axial-vector meson spectral functions [14], in this paper we include the contributions from the (axial-)vector mesons themselves to these aFRG flows. The structure of the propagators for the massive (axial-)vector fields of the effective theory is thereby fixed via current-field identities, i.e., requiring it to agree with that of analogous singleparticle or resonance contributions to the corresponding conserved currents. In particular, this implies that the massless single-particle contributions of the Stueckelberg formulation must be avoided. To implement such a structure into the FRG framework, we have to go beyond the leading order in the derivative expansion. Additional longitudinal modes are included which switch themselves off in the infrared, ensuring the transversality of the massive (axial-)vector correlations in this limit. To demonstrate the feasibility of this formulation, we compute the $\rho$ and $a_{1}$ spectral functions from the resulting aFRG flow equations at finite temperature and density, across the critical end point (CEP) in the phase diagram of the model, in parallel with the previous study in [14] but now with the fluctuations due to the massive vector and axial-vector mesons included. We identify the new contributions that arise from these fluctuations, and assess their influence on the temperature and chemical-potential-dependent pole masses, the possible signatures of a CEP and the restoration of chiral symmetry.

This paper is organized as follows: In Sec. II A we discuss the formulation of massive vector fields and verify the extraction of the vector spectral function from the imaginary part of the resulting transverse retarded propagator. After motivating and introducing the effective model in Sec. II B we show how the massive vector-meson propagators can be implemented in the FRG framework in Sec. II C. In Sec. III we then present the numerical results, first for the Euclidean parameters in Sec. III A, then the real-time results in the vacuum in Sec. III B, and finally the $\rho$ and $a_{1}$ spectral functions at finite temperature and chemical potential in Sec. IIIC. We conclude with our summary in Sec. IV. An effective Lagrangian for massive vector fields based on antisymmetric rank-2 tensors [45] is shown to lead to vector correlators of the same form in the Appendix A. Further technical details concerning the FRG flow equations and the analytic continuation procedure are provided in Appendixes B and C.

\section{THEORETICAL SETUP}

\section{A. Massive vector fields and covariant time ordering}

To describe massive vectors by fundamental fields in an effective theory is known to be problematic [46,47], if not impossible, without the Higgs mechanism. In the Proca formalism the transversality of the corresponding Green's functions is maintained only on-shell which, among other problems, leads to a pathological ultraviolet behavior. While this is fixed in the Stueckelberg formalism, one is then left with spurious massless singleparticle contributions to the vector Green's functions when restoring transversality in the Stueckelberg limit. Essentially the same is true for Nakanishi's $B$-field formalism.

There is of course no problem with massive vectors in Abelian-Higgs or Fradkin-Shenker models, for example, or in the Standard Model for that matter. However, the physical and hence gauge-invariant vectors are then necessarily described by composite fields $[48,49]$. Here we adopt a somewhat simpler approach to describe fluctuations due to (axial-)vector mesons within our FRG framework below. It starts from the fairly general point of view, describing massive vectors as single-particle contributions, which may well be composites, to the corresponding conserved vector-current correlation functions. In order to understand their spectral representations, on the other hand, it is important to remember the subtlety in defining covariant time ordering for vector or higher-rank tensor field operators [50-53].

Our brief review here follows the discussion in [46] for the simplest example of the correlation function of a conserved $U(1)$ current $j_{\mu}(x)$. As for any Feynman propagator, its causal Green's function must be covariant while the naive time-ordered product is not. One therefore defines covariant time ordering,

$$
\begin{aligned}
\left\langle T_{\operatorname{cov}} j_{\mu}(x) j_{\nu}(0)\right\rangle= & \theta\left(x^{0}\right)\left\langle j_{\mu}(x) j_{\nu}(0)\right\rangle \\
& +\theta\left(-x^{0}\right)\left\langle j_{\nu}(0) j_{\mu}(x)\right\rangle+\tau_{\mu \nu}(x),
\end{aligned}
$$

which differs from naive time ordering by a seagull term $\tau_{\mu \nu}(x)$ proportional to a delta distribution at $x=0$. Such a seagull term must occur whenever the corresponding equaltime commutators between different current components contain a Schwinger term [54]. In our example this is the case for

$$
\left.\left\langle\left[j_{0}(x), j_{i}(0)\right]\right\rangle\right|_{x^{0}=0}=\mathrm{i} \partial_{i} \delta^{3}(\vec{x}) \int_{0}^{\infty} d s \frac{\rho(s)}{s},
$$


where $\rho(s) \geq 0$ is the spectral function of the currentcurrent correlators. Together with the covariance of their causal Green's functions, the requirement that Schwinger terms are canceled from Ward identities [55] then fixes the seagull term uniquely, in the present case,

$$
\tau_{\mu \nu}(x)=\mathrm{i}\left(g_{\mu 0} g_{\nu 0}-g_{\mu \nu}\right) \delta^{4}(x) \int_{0}^{\infty} d s \frac{\rho(s)}{s},
$$

with metric and other conventions as in [46], where it is explicitly demonstrated that this leads to a spectral representation of the causal Green's function, with covariant time ordering,

$$
\begin{aligned}
& \left\langle T_{\left.\operatorname{cov} j_{\mu}(x) j_{\nu}(0)\right\rangle}\right. \\
& \quad=-\mathrm{i} \int_{0}^{\infty} d s \frac{\rho(s)}{s} \int \frac{d^{4} p}{(2 \pi)^{4}} \mathrm{e}^{-\mathrm{i} p x} \frac{p^{2} g_{\mu \nu}-p_{\mu} p_{\nu}}{p^{2}-s+\mathrm{i} \epsilon} .
\end{aligned}
$$

This is manifestly transverse and covariant as it should be, with a measure given by the semipositive spectral density $\rho(s)$. This is the spectral function of the conserved $U(1)$ current per charge squared. Assuming a (minimal) firstorder interaction $g_{v} j_{\mu} V^{\mu}$ of the current with a vector field $V_{\mu}(x)$, with coupling $g_{v}$, it is related to the vector field's spectral function $\rho_{v}(s)$ by

$$
g_{v}^{2} \rho(s)=s^{2} \rho_{v}(s) .
$$

A massive single-particle contribution of strength $Z$ in $\rho_{v}(s)$, corresponding to a stable vector meson of mass $m_{v}$, will therefore contribute to the current spectral function with a term

$$
\rho(s)=\frac{m_{v}^{4}}{g_{v}^{2}} Z \delta\left(s-m_{v}^{2}\right)+\cdots .
$$

In order to describe such a possibly composite state by a single-particle contribution to the vector-meson field $V_{\mu}$ in the spirit of vector-meson dominance, i.e., with a currentfield identity

$$
j_{\mu}(x)=\frac{m_{v}^{2}}{g_{v}} V_{\mu}(x)
$$

we therefore need to arrive at a transverse vector-meson propagator $D_{\mu \nu}^{V}$ with a single-particle contribution of the form (here still in Minkowski space),

$$
D_{\mu \nu}^{V}(p)=-\mathrm{i} \frac{Z}{m_{v}^{2}} \frac{p^{2} g_{\mu \nu}-p_{\mu} p_{\nu}}{p^{2}-m_{v}^{2}+\mathrm{i} \epsilon}+\cdots
$$

This is not of the form of a massive Proca propagator, and it differs by a factor $p^{2} / m_{v}^{2}$ from the transverse propagator that results in the Stueckelberg limit. In particular, it does therefore not come along with massless single-particle contributions. The price, however, is the poor ultraviolet behavior when viewed as the propagator of an elementary field. Although we have therefore obviously not succeeded to describe an off-shell vector meson by an elementary field, this form is still useful for our effective description of vector-meson fluctuations. The correct low-energy effective Lagrangian for a massive transverse propagator of this form, in fact, starts from describing left- and right-handed vectors in terms of (anti-)self-dual field strengths [45] which can then be reexpressed in terms of conserved fourvectors to yield propagators of the form in Eq. (8) as we describe in Appendix A.

The introduction of a regulator function $R_{k}(p)$ to suppress fluctuations of momentum modes $p<k$ in the FRG framework requires one to modify Ward identities accordingly [56]. We will therefore use an ansatz for fluctuating vector mesons which contains additional longitudinal terms that vanish with $k \rightarrow 0$ in a way such that a transverse vector-meson propagator of the form as in Eq. (8) is obtained in the infrared as explained in Sec. II C below.

In order to extract spectral functions from the results of integrating the analytically continued FRG flow equations we also need the imaginary parts of the retarded (axial-) vector propagators. This is slightly subtle for the same reasons, Schwinger and seagull terms, but the result will luckily be just as one would naively expect.

The spectral function $\rho_{v}(s)$ is originally defined from the commutator of the vector field. Via Eq. (7) this is essentially the same as that of the current $j_{\mu}(x)$ which, however, includes the Schwinger term in Eq. (2),

$$
\begin{aligned}
& \left\langle\left[V_{\mu}(x), V_{\nu}(0)\right]\right\rangle \\
& \quad=-\int_{0}^{\infty} d s \frac{s^{2} \rho_{v}(s)}{m_{v}^{4}}\left(g_{\mu \nu}+\frac{\partial_{\mu} \partial_{\nu}}{s}\right) \mathrm{i} \Delta(x ; s),
\end{aligned}
$$

written in terms of the invariant delta function

$$
\mathrm{i} \Delta\left(x ; m^{2}\right)=\int \frac{d^{4} p}{(2 \pi)^{4}} \epsilon\left(p_{0}\right) 2 \pi \delta\left(p^{2}-m^{2}\right) \mathrm{e}^{-\mathrm{i} p x} .
$$

As usual, its Fourier transform therefore essentially defines the spectral function. In particular, we obtain here,

$$
\begin{aligned}
& \int d^{4} x \mathrm{e}^{\mathrm{i} p x}\left\langle\left[V_{\mu}(x), V_{\nu}(0)\right]\right\rangle \\
& =-2 \pi \epsilon\left(p_{0}\right) \theta\left(p^{2}\right) \frac{p^{2} \rho_{v}\left(p^{2}\right)}{m_{v}^{4}}\left(p^{2} g_{\mu \nu}-p_{\mu} p_{\nu}\right) .
\end{aligned}
$$

Expressing the invariant delta function by the imaginary part of the retarded Green's function, 


$$
\mathrm{i} \Delta\left(x ; m^{2}\right)=-2 \operatorname{Im} \Delta_{R}\left(x ; m^{2}\right),
$$

we can therefore write

$$
\begin{aligned}
& \epsilon\left(p_{0}\right) \theta\left(p^{2}\right) p^{2} \rho_{v}\left(p^{2}\right)\left(p^{2} g_{\mu \nu}-p_{\mu} p_{\nu}\right) \\
& \quad=\frac{1}{\pi} \int_{0}^{\infty} d s s^{2} \rho_{v}(s)\left(g_{\mu \nu}-\frac{p_{\mu} p_{\nu}}{s}\right) \operatorname{Im} \frac{-1}{\left(p_{0}+\mathrm{i} \epsilon\right)^{2}-\vec{p}^{2}-s} .
\end{aligned}
$$

This is not quite the imaginary part of the retarded propagator corresponding to Eq. (4) yet. However, because the imaginary part of the Fourier transform of $\Delta_{R}\left(x, m^{2}\right)$ has support only at $p^{2}=m^{2}$ we can trade powers of $p^{2}$ for matching powers of $s$ in this spectral integral to write

$$
\begin{aligned}
& \epsilon\left(p_{0}\right) \theta\left(p^{2}\right) \rho_{v}\left(p^{2}\right)\left(g_{\mu \nu}-\frac{p_{\mu} p_{\nu}}{p^{2}}\right) \\
& =-\frac{1}{\pi} \operatorname{Im} \int_{0}^{\infty} d s \frac{\rho_{v}(s)}{s} \frac{p^{2} g_{\mu \nu}-p_{\mu} p_{\nu}}{\left(p_{0}+\mathrm{i} \epsilon\right)^{2}-\vec{p}^{2}-s} .
\end{aligned}
$$

This confirms that we can safely extract also a vector spectral function from the discontinuity along the cut of the transversally projected vector propagator with spectral representation as in Eq. (4), i.e., from the transverse imaginary part of a retarded vector propagator of the form

$$
D_{\mu \nu}^{T, R}(p)=\int_{0}^{\infty} d s \frac{\rho_{v}(s)}{s} \frac{p^{2} g_{\mu \nu}-p_{\mu} p_{\nu}}{\left(p_{0}+\mathrm{i} \epsilon\right)^{2}-\vec{p}^{2}-s} .
$$

\section{B. Gauged linear sigma model with quarks and the FRG}

In this section we briefly introduce the effective model we employ. For a more detailed discussion of the model we refer to $[14,31]$.

The starting point is the linear sigma model with quarks, often used as effective low-energy model for two-flavor QCD to study the chiral phase transition. It contains the isotriplet $\vec{\pi}$ and the isosinglet $\sigma$ as chiral partners in the scalar sector which are coupled to quark-antiquark fields with a Yukawa-type interaction. In these type of models one has a CEP at low temperature and large quark chemical potential, separating a crossover transition at larger temperatures from a first-order phase transition at lower ones, into a dense region of self-bound quark matter. The $\sigma$ field contains an exactly massless critical mode at the CEP. Its expectation value $\sigma_{0}$ serves as an order parameter for (spontaneous) chiral symmetry breaking and is fixed by the pion decay constant.

In order to model confinement, Polyakov-loop extensions are usually employed to suppress the contributions from quark degrees of freedom in the pressure and other bulk thermodynamic observables. Because extensions of this kind are not suited to suppress equally unphysical quark-antiquark thresholds in spectral functions, and were hence not used in [14] where this is discussed in more detail, we will not use them here either. We will accept the quark thresholds seen in Figs. 9-11 as model peculiarities. Instead of an improved modeling of confinement, one might as well replace the quarks with baryons in the future, leading to physical bayonic thresholds at correspondingly higher energies in a purely hadronic effective low-energy theory based, for example, on a chiral parity-doublet baryon-meson model [57].

Either way, vector mesons are usually introduced in such models as the gauge fields of a local flavor symmetry, first proposed by Sakurai [58] and later extended to the full chiral group to introduce the vector and axial-vector isotriplets $\vec{\rho}$ and $\vec{a}_{1}$ as the gauge fields of a local chiral symmetry $S U(2)_{L} \times S U(2)_{R}$ [59]. This idea of local gauge invariance was also the origin of the concept of VMD [10]. Alternatively, one sometimes imposes only a global chiral symmetry rather than a local one [32,60].

In this work we employ the gauged linear sigma model with quarks based on the assumption of VMD within the framework of the functional renormalization group. The idea of the FRG is to introduce a momentum scale $k$ and to integrate out fluctuations with momenta larger than this scale. To this end one defines the so-called effective average action $\Gamma_{k}$ which interpolates between the classical action $S \equiv \Gamma_{k \rightarrow \Lambda}$ at some chosen UV cutoff scale $\Lambda$ and the full quantum effective action $\Gamma_{k \rightarrow 0}$ at the infrared (IR) scale $k \rightarrow 0$. Technically this is achieved by implementing a regulator function $R_{k}$ which suppresses low-momentum fluctuations. The change of $\Gamma_{k}$ when changing the scale $k$ is described by the so-called Wetterich equation [61],

$$
\partial_{k} \Gamma_{k}=\frac{1}{2} \operatorname{Tr}\left[\partial_{k} R_{k}^{\phi}\left(\Gamma_{k}^{(2)}[\phi]+R_{k}^{\phi}\right)^{-1}\right]
$$

One hence starts with an ansatz for the effective average action $\Gamma_{k=\Lambda}$ at the UV scale and integrates out fluctuations momentum shell by momentum shell until arriving at the IR scale $\Gamma_{k \rightarrow 0}$. From suitable functional derivatives of Eq. (13) one furthermore obtains flow equations for the corresponding scale-dependent $n$-point vertex functions $\Gamma_{k}^{(n)}$.

A widely used ansatz for the Euclidean effective average action is provided by the leading order in a derivative expansion, in the literature also referred to as the local potential approximation (LPA). For the extended linear sigma model with gauged $S U(2) \times S U(2) \simeq S O(4)$ chiral symmetry, for example, this ansatz is of the following form, 


$$
\begin{aligned}
\Gamma_{k}= & \int d^{4} x\left\{\bar{\psi}\left(\not \partial-\mu \gamma_{0}+h_{s}\left(\sigma+\mathrm{i} \vec{\tau} \cdot \vec{\pi} \gamma_{5}\right)+\mathrm{i} h_{v}\left(\gamma_{\mu} \vec{\tau} \cdot \vec{\rho}_{\mu}+\gamma_{\mu} \gamma_{5} \vec{\tau} \cdot \vec{a}_{1 \mu}\right)\right) \psi+U_{k}\left(\phi^{2}\right)-c \sigma\right. \\
& \left.+\frac{1}{2}\left(D_{\mu} \phi\right)^{\dagger} D_{\mu} \phi+\frac{1}{8} \operatorname{tr}\left(V_{\mu \nu} V_{\mu \nu}\right)+\frac{1}{4} m_{v, k}^{2} \operatorname{tr}\left(V_{\mu} V_{\mu}\right)+\frac{1}{4} \lambda_{k} \operatorname{tr}\left(\partial_{\mu} V_{\mu}\right)^{2}\right\}
\end{aligned}
$$

where, compared to [14], we only added the last term with scale-dependent Stueckelberg parameter $\lambda_{k}$ for now. We will have to go beyond this leading order in the derivative expansion to accommodate propagators of the form in Eq. (8) for massive vector and axial-vector fields, however. This will be discussed in the next subsection.

As usual, the (pseudo)scalar mesons are collected in four-vectors, and the (axial-)vector mesons in skewsymmetric Hermitian matrices transforming in the adjoint $S O(4)$ representation,

$$
\begin{gathered}
\phi \equiv(\vec{\pi}, \sigma)^{T}, \\
V_{\mu} \equiv \vec{\rho}_{\mu} \cdot \vec{T}+\vec{a}_{1 \mu} \cdot \vec{T}^{5} .
\end{gathered}
$$

The six Hermitian generators $\vec{T}$ and $\vec{T}^{5}$ in the $\mathfrak{g} \mathfrak{o}(4)$ algebra are defined such that $T_{i}^{L} \equiv \frac{1}{2}\left(T_{i}-T_{i}^{5}\right)$ and $T_{i}^{R} \equiv \frac{1}{2}\left(T_{i}+T_{i}^{5}\right)$ generate the $(1,0)$ and $(0,1)$ representations of $S U(2)_{L} \times$ $S U(2)_{R}$,

$$
\begin{gathered}
{\left[T_{i}^{L}, T_{j}^{L}\right]=\mathrm{i} \epsilon_{i j k} T_{k}^{L},} \\
{\left[T_{i}^{L}, T_{j}^{R}\right]=0,} \\
{\left[T_{i}^{R}, T_{j}^{R}\right]=\mathrm{i} \epsilon_{i j k} T_{k}^{R} .}
\end{gathered}
$$

The first part in Eq. (14) describes two quark flavors with chemical potential $\mu$ plus Yukawa couplings $h_{S}$ to the (pseudo)scalar mesons and $h_{v}$ to the (axial) vectors. Then we have the $O(4)$-invariant effective potential including scalar meson self-interactions and the explicit chiral symmetry breaking term $c \sigma$. Next we have the kinetic term for the scalars coupled minimally, via a covariant derivative $\partial_{\mu}+\mathrm{i} g V_{\mu}$, with gauge coupling $g$, to the vector mesons $V_{\mu}$ whose non-Abelian field-strength tensor would be given by

$$
V_{\mu \nu}=\partial_{\mu} V_{\nu}-\partial_{\nu} V_{\mu}+\mathrm{i} g\left[V_{\mu}, V_{\nu}\right] .
$$

As in [14] we will neglect their self-interactions here, however, and only maintain the Abelian part. Then, the last three terms on the right-hand side in Eq. (14) together represent one equal Abelian Stueckelberg Lagrangian for each of the six massive vector fields described by $V_{\mu}$, related to one another by a global $S O(4)$ symmetry. This part will need further amendment as described below.

Moreover note that in a description with a fully gauged $S U(2) \times S U(2)$ local chiral symmetry and a photon coupling according to Kroll et al. [62] one would have to require $h_{v}=g / 2$ in order to be consistent with the original VMD interaction with coupling $g_{v}=g$. For a most direct comparison we instead use $h_{s}=h_{v}$ here, as was done in the previous study in Ref. [14].

Taking two functional derivatives of the Wetterich equation, Eq. (13), and using an ansatz of the from in Eq. (14) one obtains flow equations for the Euclidean $\rho$ and $a_{1}$-meson two-point functions. Without truncations this would still result in an infinite tower of equations as the flow of $\Gamma_{k}^{(n)}$ involves vertex functions up to $\Gamma_{k}^{(n+2)}$. To obtain a closed set of equations we therefore extract the $n$-point functions needed inside the flow equation for $\Gamma_{k}^{(2)}$ from the LPA ansatz of the effective action in Eq. (14). This thermodynamically consistent and symmetry-preserving scheme is self-consistent for the two-point functions only in the limit of vanishing momenta, however. Moreover, the scale-dependent three and four-point functions $\Gamma_{k}^{(3)}$ and $\Gamma_{k}^{(4)}$ are themselves momentum independent in this truncation. Self-consistent two-point functions at finite momenta, and three- and four-point functions with nontrivial internal substructures will be left as important extensions for future studies. The resulting equations are shown diagrammatically in Fig. 1, where one can already identify the kind of processes that can occur in this truncation. After the analytic continuation procedure, their real and imaginary parts basically determine the spectral functions, cf. Apppendix C.

\section{Vector-meson fluctuations}

In this section we explain how to implement propagators for single-particle contributions of the form in Eq. (8) from massive (axial) vectors, as discussed in Sec. II A, into the FRG flow equations. Since these are formulated in Euclidean spacetime, we first write the corresponding Feynman propagator in Eq. (8) as transverse Euclidean momentum-space propagator,

$$
D_{\mu \nu}^{T, \mathrm{E}}(p)=\frac{Z}{m^{2}} \frac{-p^{2}}{p^{2}+m^{2}} \Pi_{\mu \nu}^{T}(p),
$$

with transverse projector $\Pi_{\mu \nu}^{T}(p)=\delta_{\mu \nu}-p_{\mu} p_{\nu} / p^{2}$.

We also need the corresponding two-point function $\Gamma_{\mu \nu}^{(2), \mathrm{E}}(p)$ in the FRG calculation, given by the inverse of the propagator. One therefore adds a constant longitudinal part in the Stueckelberg formalism, as also done for example in $[32,60]$. The Stueckelberg Lagrangian in 


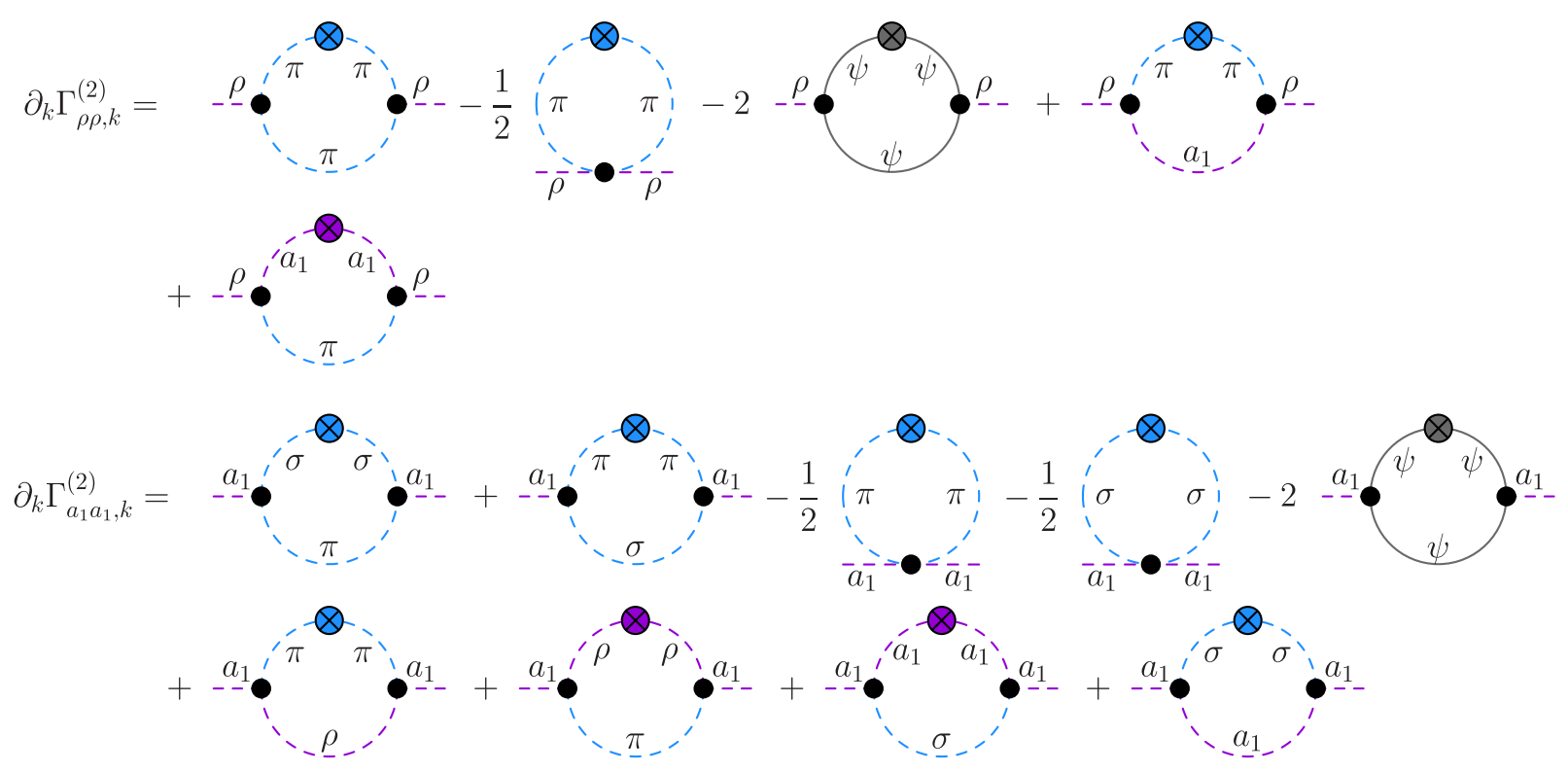

FIG. 1. Flow equations for the $\rho$ and $a_{1}$ two-point functions in diagrammatic form. Vertices are indicated by black filled dots, regulator insertions by crossed circles. The color of the lines and regulators represents the type of field: blue for scalar and pseudoscalar mesons, black for fermions and purple for vector mesons.

Eq. (14) corresponds to the following LPA-like ansatz for the massive vector two-point functions

$\Gamma_{\mu \nu, k}^{(2), \mathrm{E}}(p)=\left(p^{2}+m_{v, k}^{2}\right) \Pi_{\mu \nu}^{T}(p)+\left(\lambda_{k} p^{2}+m_{v, k}^{2}\right) \Pi_{\mu \nu}^{L}(p)$,

where $\Pi_{\mu \nu}^{L}(p)=\delta_{\mu \nu}-\Pi_{\mu \nu}^{T}(p)=p_{\mu} p_{\nu} / p^{2}$.

With such an approach, based on the Stueckelberg LPA ansatz [Eq. (14)], however, one is always left with nonvanishing unphysical longitudinal contributions of the vector-meson propagators inside the loops (see Fig. 1). In particular, these unphysical fluctuations can lead to sizable negative contributions to spectral functions and hence to positivity violations, indicating that even the Abelian gauge invariance is lost. In order to fix this problem, we modify the massive vector part of our Stueckelberg LPA ansatz for the scale-dependent effective average action in the following way: In the parts that are quadratic in the vector fields, in momentum space, we first replace the Stueckelberg LPA form of $\Gamma_{\mu \nu, k}^{(2), \mathrm{E}}$ in Eq. (22) by

$$
\begin{aligned}
\Gamma_{\mu \nu, k}^{(2), \mathrm{E}}(p)= & Z_{k}^{T}\left(p^{2}\right)\left(p^{2}+m_{v, k}^{2}\right) \Pi_{\mu \nu}^{T}(p) \\
& +\lambda_{k} Z_{k}^{L}\left(p^{2}\right)\left(p^{2}+m_{v, k}^{2} / \lambda_{k}\right) \Pi_{\mu \nu}^{L}(p),
\end{aligned}
$$

with momentum-dependent longitudinal and transverse wave function renormalizations $Z_{k}^{L}\left(p^{2}\right)$ and $Z_{k}^{T}\left(p^{2}\right)$ in addition to the scale-dependent mass and Stueckelberg parameters. In order to restore the transversality of the corresponding propagator in the infrared,

$$
p_{\mu} D_{k \rightarrow 0}^{\mu \nu}=0
$$

which is anyway the proper generalization of the corresponding Ward identity, once a regulator function $R_{k}\left(p^{2}\right)$ is introduced [56], we then choose a running Stueckelberg parameter that starts at the ultraviolet cutoff $k=\Lambda$ with some finite value $\lambda_{\Lambda}>0$ and tends to zero for $k \rightarrow 0$ in order to effectively make the longitudinal fluctuations, now with mass $m_{v, k}^{2} / \lambda_{k}$, infinitely heavy in the infrared. One simple choice that does the job is

$$
\lambda_{k}=\frac{k^{2}}{\Lambda^{2}}, \quad \text { such that } \lambda_{\Lambda}=1, \quad \lambda_{k} \stackrel{k \rightarrow 0}{\longrightarrow} 0 .
$$

The specific functional form of $\lambda_{k}$ is to a large extent arbitrary here, as long as it starts from some finite value $\lambda_{\Lambda}$ at the cutoff scale $\Lambda$ and vanishes suitably rapidly with $k \rightarrow 0$ in the infrared, so that the longitudinal modes decouple in this limit. The form in Eq. (25) represents arguably the simplest choice with these properties. Moreover, as further discussed below, we have verified numerically that our results become totally insensitive to the longitudinal fluctuations for sufficiently small ultraviolet start values $\lambda_{\Lambda}$ in the first place.

In addition, we require the longitudinal renormalization function to cancel the explicit factor $\lambda_{k}$ in front of the longitudinal term. A simple way to realize this is to set

$$
\lambda_{k} Z_{k}^{L}\left(p^{2}\right)=Z_{k}^{T}\left(p^{2}\right)
$$

which we assume to be independent of the running Stueckelberg parameter $\lambda_{k}$. Note that assuming $Z_{k}^{L}=Z_{k}^{T}$ 
and both to be independent of $\lambda_{k}$ instead, would essentially lead back to the Proca propagator for $\lambda_{k} \rightarrow 0$ in the infrared (i.e., up to the factor $Z_{k}^{T}$ ). In the present setup on the other hand, longitudinal and transverse components start out equally at the ultraviolet cutoff scale for $k=\Lambda$ so that the two-point function is altogether proportional to $\delta_{\mu \nu}$ (as in Feynman gauge). Relative to the transverse mass, the longitudinal mass becomes heavier and heavier and the unphysical longitudinal fluctuations switch themselves off automatically during the flow.

Finally, for the transverse part to correctly model the single-particle contribution to the vector correlator (8), cf. Eq. (21) with scale-dependent mass $m_{v, k}$ and strength $Z_{k}$, all we now have left to do is to set

$$
Z_{k}^{T}\left(p^{2}\right)=-Z_{k}^{-1} m_{v, k}^{2} / p^{2} \equiv-m_{0, k}^{2} / p^{2},
$$

with an independent mass parameter $m_{0, k}^{2}=m_{v, k}^{2} / Z_{k}$ which for $Z_{k}<1$ we expect to be larger than the scale-dependent (pole) mass of the vector meson $m_{v, k}^{2}$, in particular for $k \rightarrow 0$ in the infrared. We will start the flow with the boundary condition at the ultraviolet cutoff $k=\Lambda$ with $Z_{\Lambda}=1$, corresponding to the full spectral strength initially contained in this single-particle contribution.

Note that the somewhat ambiguous details in the treatment of the unphysical longitudinal fluctuations are irrelevant here: Just as the vector-meson mass parameter, the longitudinal mass starts out at a rather large initial value of about

$$
m_{l, \Lambda}=m_{v, \Lambda} \approx 1 \mathrm{GeV}
$$

as compared to a UV cutoff for which we typically use $\Lambda=1.5 \mathrm{GeV}$. Because the longitudinal mass

$$
m_{l, k}=\frac{\Lambda}{k} m_{v, k}
$$

increases rapidly with lower $k$ from there on, these longitudinal fluctuations are strongly suppressed during the flow. In principle, their suppression can be further controlled by the initial value of the Stueckelberg parameter $\lambda_{\Lambda}$. One then verifies that the results are in fact independent of this parameter for sufficiently small $\lambda_{\Lambda}$. Our choice of $\lambda_{\Lambda}=1$ seems rather natural but is by no means mandatory, it simply turns out to be sufficiently small for the parameters used here at least at low temperatures. For higher temperatures, e.g., for the results presented in the next section with $T=100 \mathrm{MeV}$ and above, we in fact observe that longitudinal fluctuations can occasionally still produce small spurious contributions to capture processes during the flow, when their initial mass is not large enough for $\lambda_{\Lambda}=1$. In such cases we simply reduce $\lambda_{\Lambda}$ further, until we observe no noticeable dependence on $\lambda_{\Lambda}$ anymore.
In contrast, we have been very careful in modeling the transverse fluctuations to correctly describe the singleparticle contributions to the full (axial-)vector correlators, including momentum- and field-independent wave function renormalization in the form of the scale-dependent strength $Z_{k}$. This treatment of the (axial-)vector fluctuations thus in this sense parallels what has been called the LPA truncation for the (pseudo)scalar sector in the literature.

Choosing a transverse and longitudinal regulator functions of the same form,

$$
R_{\mu \nu, k}^{T, L}(p)=Z_{k}^{T, L}\left(p^{2}\right) k^{2} r_{k}(p) \Pi_{\mu \nu}^{T, L}(p),
$$

with a suitable dimensionless regulator function $r_{k}(p)$, and using Eqs. (25)-(27) in Eq. (23), the ansatz for the scaledependent vector propagator with regulator becomes

$$
\begin{aligned}
D_{\mu \nu, k}^{\mathrm{E}}(p) \equiv & \left(\Gamma_{k}^{(2), \mathrm{E}}(p)+R_{k}(p)\right)_{\mu \nu}^{-1} \\
= & \frac{-p^{2}}{m_{0, k}^{2}\left(p^{2}+k^{2} r_{k}(p)+m_{v, k}^{2}\right)} \Pi_{\mu \nu}^{T}(p) \\
& \quad-\frac{p^{2}}{m_{0, k}^{2}\left(p^{2}+k^{2} r_{k}(p)+\frac{\Lambda^{2}}{k^{2}} m_{v, k}^{2}\right)} \Pi_{\mu \nu}^{L}(p) .
\end{aligned}
$$

For $k \rightarrow 0$ we recover Eq. (24), and the transverse part reduces to the desired single-particle contribution of a massive vector state as in Eq. (21). The flow of the mass parameter $m_{0, k}^{2}=m_{v, k}^{2} / Z_{k}$ is in general different from that of the LPA vector-meson mass $m_{v, k}^{2}$, of course. As mentioned above, we will assume their initial values to be the same at the UV cutoff, with $Z_{\Lambda}=1$, i.e.,

$$
m_{0, \Lambda}^{2}=m_{v, \Lambda}^{2}
$$

Due to the fluctuations in the interacting theory one then expects $Z_{k}<1$ and, with $\lambda_{k}=k^{2} / \Lambda^{2}$, therefore the overall ordering

$$
m_{l, k}^{2}>m_{0, k}^{2}>m_{v, k}^{2}, \quad \text { for } k<\Lambda .
$$

This behavior is confirmed explicitly in the numerical calculations as described in the next section, Sec. III A.

As mentioned above, the LPA ansatz in Eq. (31) is used to describe the fluctuations due to the single-particle contributions of massive vectors on the right-hand side of the FRG flow equations in Fig. 1, in our present truncation. The result of the integrated flow on the lefthand side yields the corresponding full two-point functions including widths and various thresholds whose transverse parts will have a spectral representation as in Eq. (12). In a fully self-consistent calculation one would have to feed these back into the flow equations, recompute and iterate until convergence [42]. As in our previous studies 
$[14,37,38]$ here we perform the first step in such an approach, thus neglecting the fluctuations due to the continuous contributions from the two-point functions inside the flow.

\section{NUMERICAL RESULTS}

\section{A. Euclidean FRG flow and mass parameters}

In this section we discuss the numerical procedure for solving the Euclidean flow equations and the resulting flow of the Euclidean (curvature) mass parameters. Since the setting used here in wide parts parallels that of Ref. [14] we keep the discussion brief and refer to this reference as well as our Appendix B for further details.

We start with solving the flow equation for the effective potential $U_{k}\left(\phi^{2}\right)$ which contains scalars and pseudoscalars as well as quarks and antiquarks as fluctuating fields. Because the Euclidean mass parameters of the (axial-) vector mesons are relatively heavy, of the order of the UV cutoff $\Lambda=1500 \mathrm{MeV}$, they are expected to contribute very little to the Euclidean flow of the effective potential and are therefore neglected. The flow equation for $U_{k}\left(\phi^{2}\right)$ is then solved with standard procedures by discretizing its argument in field space, and using the following simple form of the linear sigma model in the symmetric phase at the UV scale $\Lambda$ as initial condition,

$$
U_{k=\Lambda}\left(\phi_{i}^{2}\right)=b_{1} \phi_{i}^{2}+b_{2} \phi_{i}^{4} .
$$

Storing the $k$-dependent effective potential, we solve the flow equations for the vector-meson mass parameters $m_{v, k}^{2}$ and $m_{0, k}^{2}$ next, see Appendix B for more details. With the parameters listed in Table I (set 1), we obtain the following values for the chiral order parameter $\sigma_{0}$ and the Euclidean mass parameters at the IR scale of $k=40 \mathrm{MeV}$,

$$
\begin{array}{rlrl}
\sigma_{0} & =93.0 \mathrm{MeV}, & m_{\sigma}=557.1 \mathrm{MeV}, \\
m_{\pi} & =140.4 \mathrm{MeV}, \quad m_{\psi}=300.0 \mathrm{MeV}, \\
m_{\rho} & =868.1 \mathrm{MeV}, & m_{a_{1}}=1363.1 \mathrm{MeV}, \\
m_{0} & =1294.3 \mathrm{MeV} . &
\end{array}
$$

The first four of the UV parameters in Table I thereby determine the infrared properties of the effective potential, such as the pion mass and decay constant as well as the

TABLE I. Different UV parameter sets resulting in roughly the same pole masses, close to the physical ones of $\rho$ and $a_{1}$, all with the same quark-meson model parameters.

\begin{tabular}{lcccccl}
\hline \hline Set No. & $b_{1}\left(\Lambda^{2}\right)$ & $b_{2}$ & $c\left(\Lambda^{3}\right)$ & $h_{s}=h_{v}$ & $g$ & $m_{v, \Lambda}(\Lambda)$ \\
\hline 1 & 0.381 & 0.2 & $0.5401 \times 10^{-3}$ & 3.226 & 11.3 & 0.7067 \\
2 & & & & & 11.8 & 0.684 \\
3 & & & & & 10.5 & 0.74 \\
\hline \hline
\end{tabular}

quark and sigma meson mass parameters in the usual way. The values of these four parameters are not adjusted when solving the flow equations for either the Euclidean vectormeson mass parameters or their aFRG flow equations which do not in turn affect the effective potential. The first four parameters of Table I are therefore the same for all three parameter sets.

Note that the mesonic mass parameters in Eq. (35) are not directly the physical meson masses. They are determined from the zero-momentum limit of the respective Euclidean two-point functions $\Gamma^{(2), E}(p)$. For the (pseudo) scalars they agree with the corresponding curvatures in the $\pi$ and $\sigma$ directions of the effective potential in our thermodynamically consistent and symmetry-preserving truncation scheme.

The analogous Euclidean masses of vector and axialvector meson, on the other hand, then essentially result from tuning their coupling $g$ and UV mass parameter $m_{v, \Lambda}$ so that the corresponding pole masses $m_{\rho}^{p}$ and $m_{a_{1}}^{p}$ assume the approximate mass values of the physical $\rho(770)$ and the $a_{1}(1260)$ mesons. Since the $\rho$ and $a_{1}$ are resonances, we estimate their pole masses from the zeros of the real parts of the respective retarded two-point functions, $\Gamma_{\rho / a_{1}}^{(2), R}(p)$, which is a fairly good approximation as long as the widths of the resonances, i.e., the imaginary parts of $\Gamma_{\rho / a_{1}}^{(2), R}$ in the resonance region, are sufficiently small. In our present qualitative study we are content with this approximation and obtain the pole masses listed in Table II which are all reasonably close to the physical masses of $\rho$ and $a_{1}$ for our representative UV parameters of Table I. For a more precise determination of masses and widths one would have to study the analytic structure of $\Gamma_{\rho / a_{1}}^{(2), R}$ and look for the resonance poles on the unphysical second Riemann sheet, see e.g., Refs. $[63,64]$.

The $k$ flow of the Euclidean masses and of the mass parameter $m_{0, k}$, all evaluated at the $k$-dependent minimum $\sigma_{0, k}^{2}$ of $U_{k}\left(\phi^{2}\right)$, is plotted in Fig. 2. Starting at the UV scale where chiral symmetry is restored, the masses of the chiral partners $\pi-\sigma$ and $\rho-a_{1}$ are degenerate, the quark mass has its very small bare value. Taking fluctuations into account by successively lowering the scale $k$, the mass parameter $m_{0, k}$ immediately splits from its counterpart $m_{v, k}$ because their

TABLE II. Pole masses of (axial-)vector mesons for the parameter sets of Table I compared to the estimates for $\rho(770)$ and $a_{1}(1260)$ from the Review of Particle Properties in Particle Data Group (PDG) (2019).

\begin{tabular}{lcc}
\hline \hline Set No. & $m_{\rho}^{p}(\mathrm{MeV})$ & $m_{a_{1}}^{p}(\mathrm{MeV})$ \\
\hline 1 & 776.3 & 1242.6 \\
2 & 774.9 & 1266.2 \\
3 & 770.2 & 1258.6 \\
PDG & $775.26 \pm 0.25$ & $1230 \pm 40$ \\
\hline \hline
\end{tabular}




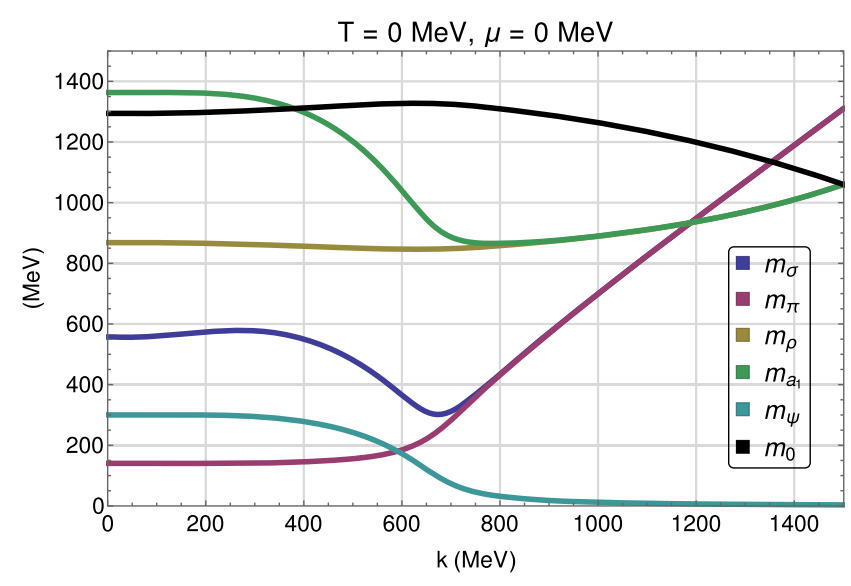

FIG. 2. Flow of the Euclidean mass parameters in Eq. (35) with the $\mathrm{RG}$ scale $k$ in the vacuum (parameter set 1 ).

flow is not independent but in fact opposite in sign. In particular, one has

$$
\frac{\partial_{k} m_{0, k}^{2}}{m_{0, k}^{2}}=-\frac{\partial_{k} m_{v, k}^{2}}{m_{v, k}^{2}},
$$

cf. Appendix B. Moreover, the relation $m_{0, k}^{2} \geq m_{v, k}^{2}$ discussed in Sec. IIC with $m_{0, \Lambda}^{2}=m_{v, \Lambda}^{2}$ therefore holds by construction whenever the flow of the vector-meson mass parameter $m_{v, k}$ is predominantly negative as in Fig. 2.

Lowering the scale further, spontaneous chiral symmetry breaking sets in, giving rise to an increase of the chiral order parameter $\sigma_{0, k}$. The quark acquires its dynamically generated constituent mass, and the masses of the chiral partners split up. At the IR scale we then arrive at the values listed in Eq. (35).

\section{B. Spectral functions in the vacuum}

In this subsection we now explicitly demonstrate that the formalism presented in Sec. IIC yields positive and physically meaningful contributions to the vector and axial-vector meson spectral functions.

Based on the Euclidean $k$ flow as input, from which all scale-dependent parameters are determined, we employ our standard analytic continuation procedure as outlined in Appendix $\mathrm{C}$ to obtain the aFRG flow equations for the retarded two-point functions $\Gamma_{\rho / a_{1}}^{(2), R}(\omega)$, here at vanishing spatial momentum $\vec{p}$, for the $\rho$ and $a_{1}$ mesons from the diagrams shown in Fig. 1.

The spectral function as a function of the external frequency $\omega$ at vanishing external spatial momentum $|\vec{p}|=$ $0 \mathrm{MeV}$ is then given by the discontinuity along the cut as verified explicitly for the transversally projected vector propagator at the end of Sec. II A, which can be extracted from the real and imaginary parts of $\Gamma_{\rho / a_{1}}^{(2), R}(\omega)$ as usual by

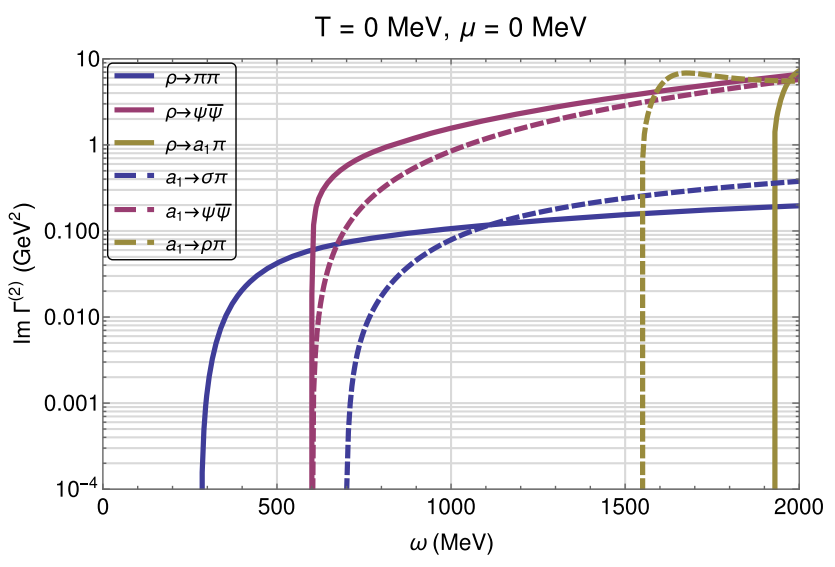

FIG. 3. Imaginary parts of the retarded two-point functions of the $\rho$ (solid lines) and $a_{1}$ meson (dashed lines) for every process separately as a function of external frequency $\omega$ (evaluated at the fixed infrared minimum $\sigma=\sigma_{0}$ using the parameters of set 2).

$$
\rho(\omega)=\frac{1}{\pi} \frac{\operatorname{Im} \Gamma^{(2), R}(\omega)}{\left(\operatorname{Re} \Gamma^{(2), R}(\omega)\right)^{2}+\left(\operatorname{Im} \Gamma^{(2), R}(\omega)\right)^{2}} .
$$

In Fig. 3 we show the imaginary parts of $\Gamma_{\rho / a_{1}}^{(2), R}(\omega)$ for every process separately. Since there are no in-medium capture processes in the vacuum, all thresholds indicate decays of off-shell (axial-)vector mesons $\rho$ and $a_{1}$ into the particle pairs as labeled in the figure, which can also be inferred from Fig. 1. In the present truncation, with only the single-particle contributions on the right-hand side of the aFRG flow equations, the positions of the thresholds are still determined by this input, i.e., by the Euclidean mass parameters at the IR scale. In a self-consistent solution [42] these thresholds will eventually be determined by the resulting physical pole masses, as they should be of course, possibly smeared out for resonances. Decays involving $\sigma$ mesons in the final state for example will then lead to two additional pions, such as $a_{1} \rightarrow \pi+\sigma$ below which will then contribute to the decay $a_{1} \rightarrow 3 \pi$.

In the spirit of the grid-code technique, the $k$-dependent input for the aFRG flow equations for real and imaginary parts of $\Gamma^{(2), R}(\omega)$ is thereby normally evaluated at the fixed value of the $\sigma$-field variable that corresponds to the minimum of the effective potential at $\sigma=\sigma_{0}=93 \mathrm{MeV}$ in the infrared. For the (axial-)vector mesons this results in mass parameters $m_{\rho}$ and $m_{a_{1}}$ that are considerably larger than those in Eq. (35) obtained from the $k$-dependent minimum $\sigma_{0, k}$ which further enhances the deviations of the two-particle thresholds from their physically expected values.

In the present setup, for example, an off-shell $\rho$ meson can decay into an $a_{1}+\pi$ pair, if the energy constraint is fulfilled, i.e., for $\omega \geq m_{a_{1}}+m_{\pi} \approx 1925.7 \mathrm{MeV}$, here with a large mass parameter $m_{a_{1}}=1785.3 \mathrm{MeV}$. Analogously, the decay $a_{1} \rightarrow \rho+\pi$ starts $\omega \geq m_{\rho}+m_{\pi} \approx 1548.6 \mathrm{MeV}$ 


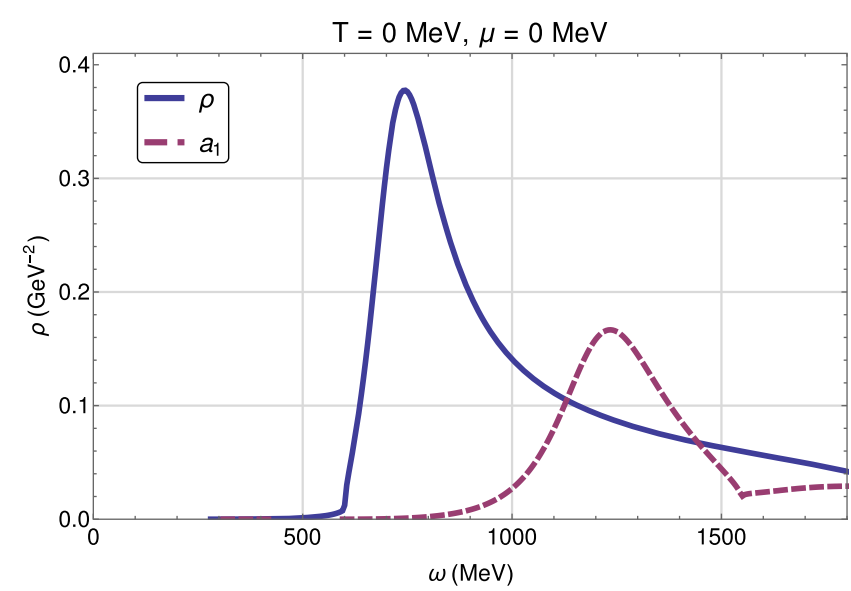

FIG. 4. Vacuum spectral function of the $\rho$ (solid blue) and $a_{1}$ meson (dashed red) as a function of external frequency $\omega$ (evaluated at fixed $\sigma=\sigma_{0}$, parameters of set 2).

with an Euclidean mass parameter $m_{\rho}=1408.2 \mathrm{MeV}$ that is also much larger than the corresponding pole mass.

Because of the necessity to suppress capture processes involving spurious longitudinal vector components at higher temperatures, as relevant in the next subsection, we have reduced $\lambda_{\Lambda}$ by about an order of magnitude here already, to further increase the longitudinal masses as mentioned in Sec. IIC. Readjusting the values of the coupling $g$ and UV mass parameter $m_{v, \Lambda}$, by using set 2 instead of set 1 here, then restores the physical pole masses of $\rho$ and $a_{1}$ again, cf. Table II. While this would not be necessary at low temperatures, we avoid changing the UV parameters at higher temperatures in this way.

The curvature masses of pion and sigma meson are the same as in Eq. (35) and so is the quark mass. The decay of a $\rho$ into pion pair therefore starts at a reasonable threshold of $280.8 \mathrm{MeV}$ here (and $a_{1} \rightarrow \sigma+\pi$ at $697.5 \mathrm{MeV}$ ). The decays into quark-antiquark pairs starting at $600 \mathrm{MeV}$ are not suppressed since there is no confinement in our model (a simple Polyakov-loop enhancement of the model will not do the job). However, all processes give positive contributions and therefore lead to positive (axial-)vector-meson spectral functions.

Putting all contributions for the real and imaginary parts of $\Gamma^{(2), R}$ together gives the vacuum spectral functions of the $\rho$ and $a_{1}$ mesons shown in Fig. 4. The peaks are in both cases located at the pole masses where the possible decays lead to rather broad structures in both spectral functions.

A rather simple improvement of the unphysically large two-particle thresholds involving decays into $\rho$ and $a_{1}$ can be obtained by giving up the unified treatment of (pseudo) scalar and (axial-)vector mesons in the following way: We use the Euclidean input evaluated at the $k$-dependent minimum $\sigma_{0, k}$ of the scale-dependent effective potential as we did for the flow of the mass parameters in the last subsection, when solving the aFRG flow equations for the $\rho$

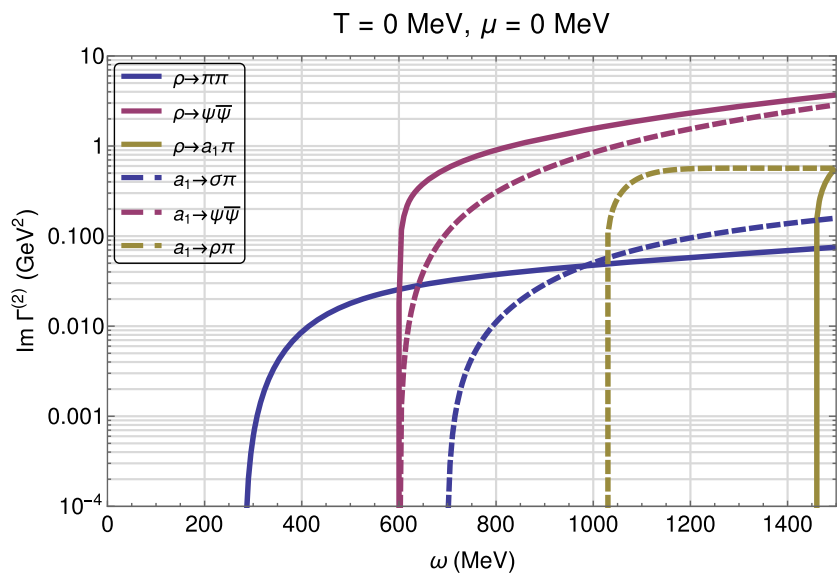

FIG. 5. Imaginary parts of the retarded two-point functions of $\rho$ (solid) and $a_{1}$ (dashed) over $\omega$ as in Fig. 3, but here evaluated at the scale-dependent $\sigma=\sigma_{0, k}$ using the parameters of set 3 .

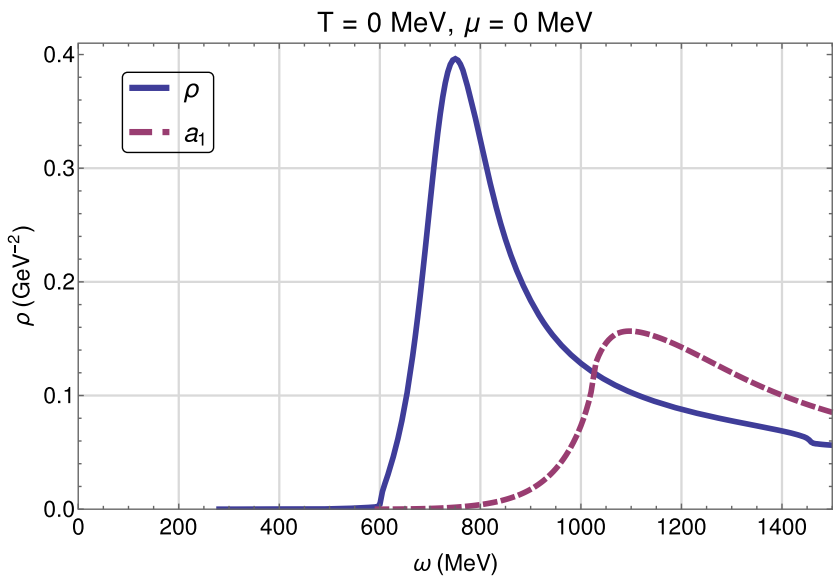

FIG. 6. Vacuum spectral functions of $\rho$ (solid) and $a_{1}$ (dashed) over $\omega$ as in Fig. 4, but here evaluated at the scale-dependent $\sigma=\sigma_{0, k}$ using the parameters of set 3 .

and $a_{1}$ two-point functions $\Gamma_{\rho / a_{1}}^{(2), R}(\omega)$. For the scale-dependent effective potential itself, on the other hand, we stick to the grid technique in the field variable $\phi^{2}$, to include the order-parameter fluctuations due to collective excitations in the $\sigma-\pi$ system as we have been doing so far.

Because this changes the pole masses of $\rho$ and $a_{1}$, we have to change their coupling $g$ and UV mass parameter $m_{v, \Lambda}$ once again to compensate for this, which is achieved here by using the parameters of set 3 in Table I. The imaginary parts of $\Gamma_{\rho / a_{1}}^{(2), R}(\omega)$ and the $\rho$ and $a_{1}$ spectral functions that result from this procedure are shown in Figs. 5 and 6.

While there are no qualitative changes as compared to the corresponding previous results in Figs. 3 and 4, with the Euclidean mass parameters $m_{\rho}=885.5 \mathrm{MeV}$ and $m_{a_{1}}=1316.2 \mathrm{MeV}$, the thresholds of $a_{1} \rightarrow \rho+\pi$ and $\rho \rightarrow$ $a_{1}+\pi$ have now moved down to $m_{\rho}+m_{\pi} \approx 1025.9 \mathrm{MeV}$ 
and $m_{a_{1}}+m_{\pi} \approx 1456.6 \mathrm{MeV}$, respectively. They are still not at the corresponding sums of pole masses, but the differences are considerably reduced by this method of evaluating the Euclidean input for the aFRG calculations of the $\rho$ and $a_{1}$ correlators at the scale-dependent minimum $\sigma_{0, k}$ of the effective potential. In contrast to the decays involving (axial) vectors, this modification has no effect on quark-antiquark thresholds or those involving only decays into $\sigma$ and $\pi$, because the relevant (curvature) masses are the same in the infrared.

\section{In-medium results}

In order to study the in-medium behavior of the $\rho$ - and $a_{1}$-meson spectral functions across the entire phase diagram of the model, the present setup is straightforwardly extended to finite temperature and quark chemical potential. In the present subsection all calculations are based on the scale-dependent Euclidean input evaluated at the fixed value in field space corresponding to the infrared minimum at $\sigma_{0}=93 \mathrm{MeV}$ again, and with the parameters of set 2 in Table I.

Because the Euclidean mass parameters determine the thresholds of all processes, we first look at their $T$ and $\mu$ dependencies which are qualitatively the same as in [14] where the corresponding phase diagram is shown as well.

In Fig. 7 we show the Euclidean mass parameters as functions of temperature at vanishing chemical potential. The qualitative behavior roughly resembles that of the scale-dependent mass parameters, as obtained by the successive inclusion of quantum fluctuations, at zero temperature in Fig. 2.

With spontaneously broken chiral symmetry in the vacuum, the zero-temperature masses of the chiral partners are split up, and the quarks are massive, here with a constituent mass of $300 \mathrm{MeV}$. The $T=0$ values of all mass parameters in Fig. 7 agree with those determining the thresholds in Fig. 3. Increasing the temperature, chiral symmetry gets gradually restored as indicated by the melting of the order parameter $\sigma_{0}(T)$ (and so of the quark mass $\left.m_{\psi}=h_{s} \sigma_{0}\right)$. At temperatures beyond the crossover

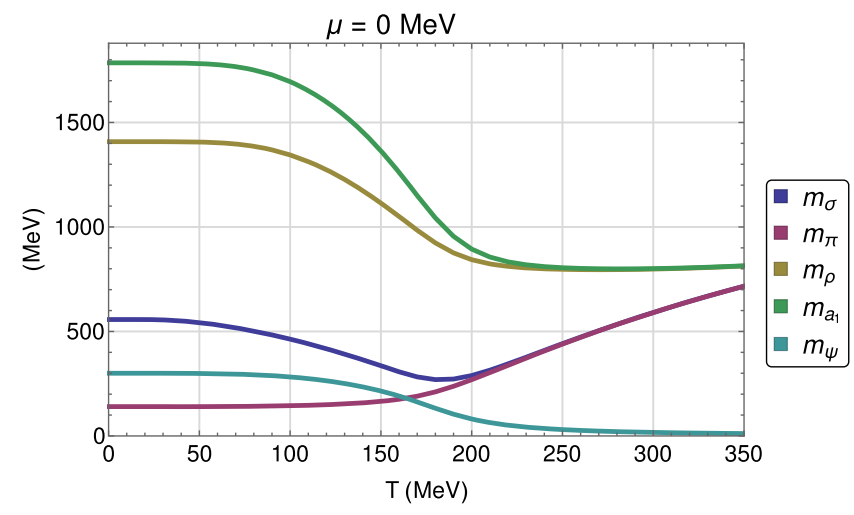

FIG. 7. Euclidean mass parameters over temperature at vanishing chemical potential. region the mass parameters of the chiral partners become rapidly degenerate.

The chemical-potential dependence of these Euclidean masses, at a fixed temperature of $T=10 \mathrm{MeV}$ which is approximately that of the CEP with our model parameters, is shown in Fig. 8. We observe that they remain constant over a wide range of $\mu$, which might seem curious at first, just as the dog that did nothing in the nighttime in The Adventure of Silver Blaze [65], and which is due the temperature being so low here. At $\mu \approx 298 \mathrm{MeV}$, however, all masses except that of the pion drop abruptly. The sigma becomes almost massless indicating the proximity of the critical end point which represents a second-order phase transition with $Z_{2}$ universality where only the correlation length of the $\sigma$ field diverges. For very large $\mu$ we again see the degeneracy of the masses of the chiral partners now reflecting the gradual restoration of chiral symmetry with density inside the high-density phase (here of self-bound quark matter).

We are now ready to discuss the temperature and chemical-potential dependence of the spectral functions of $\rho$ and $a_{1}$ mesons shown in Fig. 9. In the present setup, the following timelike decay and capture processes are possible for an off-shell $\rho^{*}$ vector meson,

$$
\begin{array}{ll}
\rho^{*} \rightarrow \psi+\bar{\psi}, & \rho^{*} \rightarrow \pi+\pi, \\
\rho^{*} \rightarrow a_{1}+\pi, & \rho^{*}+\pi \rightarrow a_{1},
\end{array}
$$

and for an off-shell $a_{1}^{*}$ axial-vector meson,

$$
\begin{array}{ll}
a_{1}^{*} \rightarrow \psi+\bar{\psi}, & a_{1}^{*}+\sigma \rightarrow \pi, \\
a_{1}^{*} \rightarrow \pi+\sigma, & a_{1}^{*}+\pi \rightarrow \sigma, \\
a_{1}^{*} \rightarrow \rho+\pi, & a_{1}^{*}+\pi \rightarrow \rho, \\
a_{1}^{*} \rightarrow a_{1}+\sigma, & a_{1}^{*}+\sigma \rightarrow a_{1},
\end{array}
$$

where each particular process is possible only if it is energetically allowed, i.e., a decay process only for energies $\omega$ in the off-shell meson correlator with

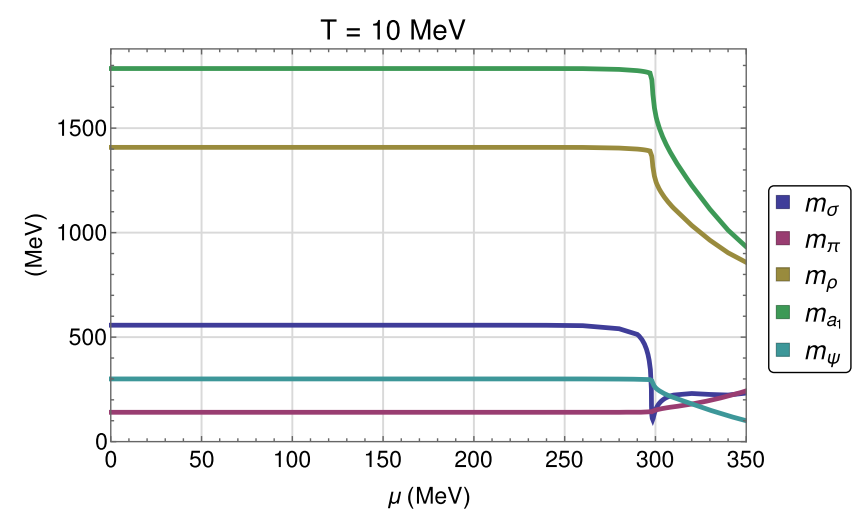

FIG. 8. Euclidean mass parameters over chemical potential at $T=10 \mathrm{MeV}$, approximately across the CEP. 

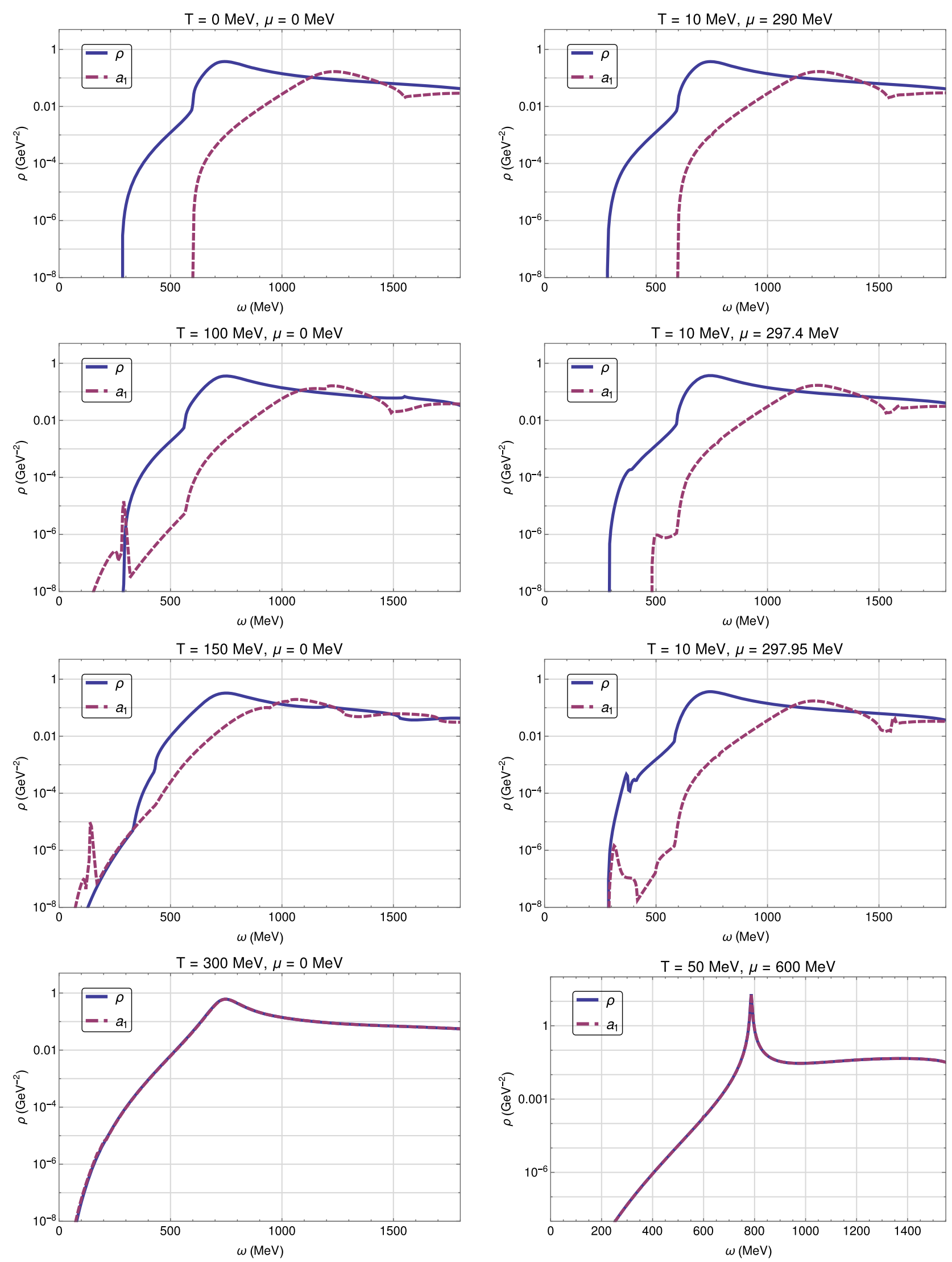

FIG. 9. In-medium spectral functions of the $\rho$ (solid blue) and $a_{1}$ meson (dashed red) as a function of external frequency $\omega$ for increasing temperature at $\mu=0 \mathrm{MeV}$ (left column) and for increasing chemical potential close to the CEP at $T=10 \mathrm{MeV}$ (right column) plotted in logarithmic scales. 
$\omega \geq m_{\alpha}+m_{\beta}$, and a capture process for $\omega+m_{\alpha} \leq m_{\beta}$ which requires $m_{\beta}>m_{\alpha}$ in addition. We have therefore not listed here the capture processes $\rho^{*}+a_{1} \rightarrow \pi, a_{1}^{*}+\rho \rightarrow \pi$ and $a_{1}^{*}+a_{1} \rightarrow \sigma$ where this never occurs, while it does happen that $m_{\sigma}<m_{\pi}$ very close to the critical end point as seen in Fig. 8.

In the vacuum only the decay processes contribute, cf. Fig. 3, and thus the starting thresholds are given by the decays into the light (pseudo)scalar mesons in both spectral functions. Here, essentially the only new feature as compared to the previous study in [14] is the decay $a_{1} \rightarrow$ $\rho+\pi$ as discussed in the previous subsection. Since the decays into quark-antiquark pairs yield rather large contributions, cf. Ref. [14], both spectral function always broaden in all $T$ and $\mu$ cases for $\omega \geq 2 m_{\psi}$. However, with increasing temperature (from top to bottom in the left column of Fig. 9) we see that the various capture processes start to contribute, giving rise to an increase in both spectral functions especially at low external frequencies, where in the $a_{1}$ spectral function the van Hove peak appears that was already observed in [14]. At $T=300 \mathrm{MeV}$ the masses of the chiral partners are degenerate and the quarks become the lightest degrees of freedom leading to broad and fully degenerate spectral functions of $\rho$ and $a_{1}$ mesons as expected in the chirally restored phase.

The most noticeable differences with respect to the previous results in [14] are the new capture processes involving vector mesons, i.e., $\rho^{*}+\pi \rightarrow a_{1}, a_{1}^{*}+\pi \rightarrow \rho$ and $a_{1}^{*}+\sigma \rightarrow a_{1}$ which fill up the gap between capture and decay processes when only the light (pseudo)scalars are involved. This is demonstrated in Figs. 10 and 11 where we plot the individual imaginary parts of the $\rho$ and the $a_{1}$ twopoint function separately that altogether contribute to their spectral functions at $T=150 \mathrm{MeV}$ as an example.

Turning on the chemical potential (as done in the right column of Fig. 9), when the low-temperature system at

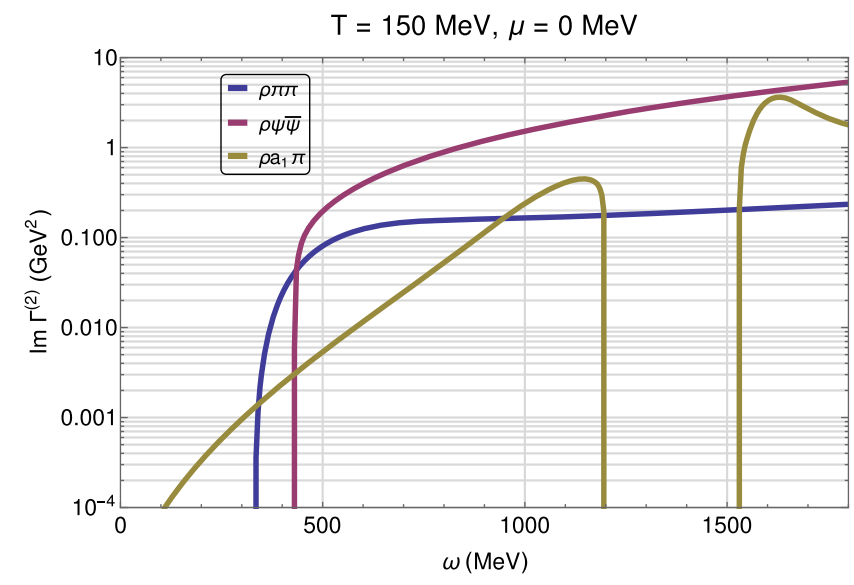

FIG. 10. Imaginary parts of the retarded two-point function of the $\rho$ meson for every process separately as a function of external frequency $\omega$ at $T=150 \mathrm{MeV}$ (evaluated at fixed IR minimum $\sigma=\sigma_{0}$ with the parameters of set 2).

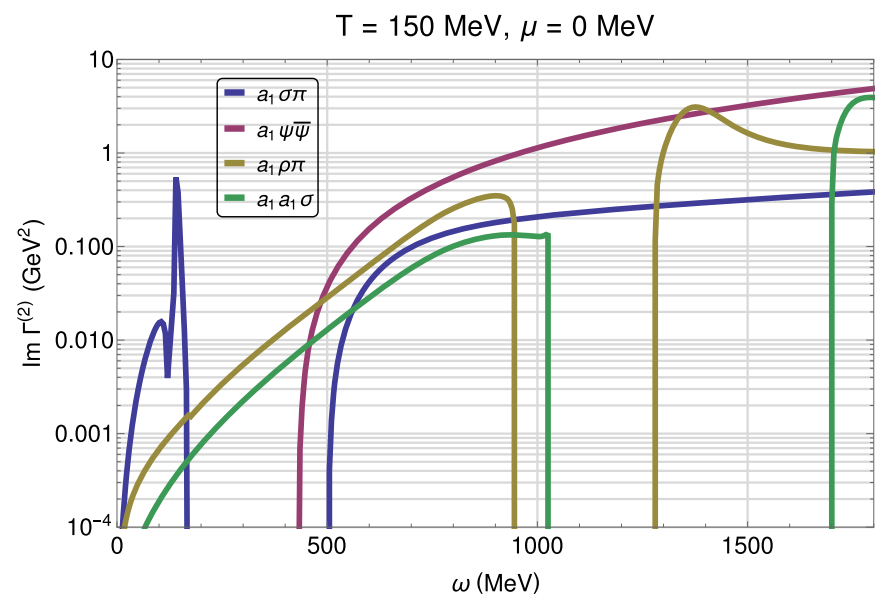

FIG. 11. Imaginary parts of the retarded two-point function of the $a_{1}$ meson for every process separately as a function of external frequency $\omega$ at $T=150 \mathrm{MeV}$ (evaluated at fixed IR minimum $\sigma=\sigma_{0}$ with parameters of set 2).

$T=10 \mathrm{MeV}$ eventually starts to react, beyond $\mu \simeq 290 \mathrm{MeV}$, we observe modifications in the $a_{1}$-meson spectral function close to the critical end point located at $\mu_{\mathrm{CEP}} \approx 298 \mathrm{MeV}$. While the $\rho$-meson spectral function remains qualitatively unchanged, the dropping threshold for $a_{1}^{*} \rightarrow \pi+\sigma$ and the related peak in the $a_{1}$ spectral function are due to the dropping sigma mass in this critical region and can thus serve as a signature for the CEP. As compared to the previous study in [14] this signal got somewhat washed out by the additionally possible processes, unfortunately, but the fact that it is robust enough to still be visible seems at least encouraging for further studies. At large $\mu$ we again observe the full degeneracy of both spectral functions with gradual chiral symmetry restoration inside the high-density phase.

To summarize, the modifications in the thermal medium are once more exemplified in Fig. 12 where the spectral

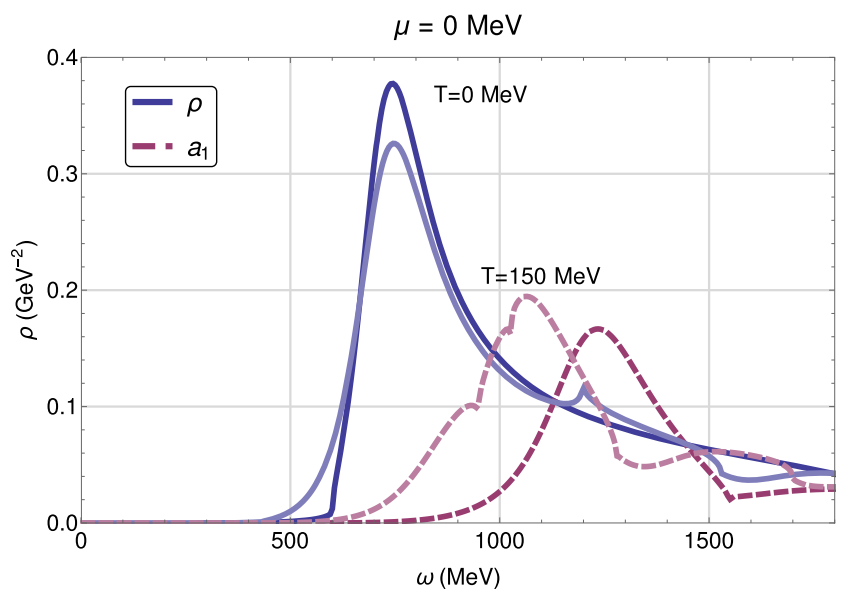

FIG. 12. Spectral functions of the $\rho$ (solid blue) and $a_{1}$ meson (dashed red) as a function of external frequency $\omega$ for $T=$ $0 \mathrm{MeV}$ (dark) and $T=150 \mathrm{MeV}$ (light). 


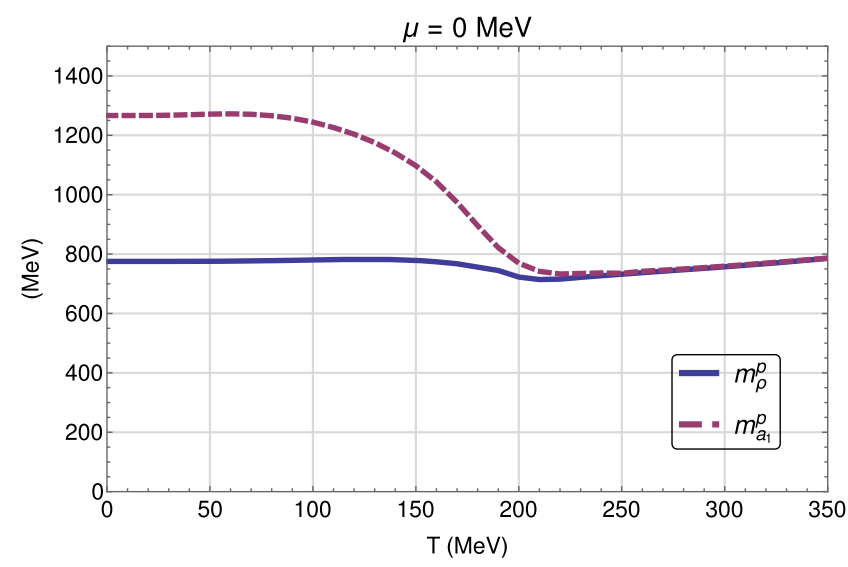

FIG. 13. Temperature dependence of the pole masses of $\rho$ (solid blue) and $a_{1}$ (dashed red) meson at $\mu=0$.

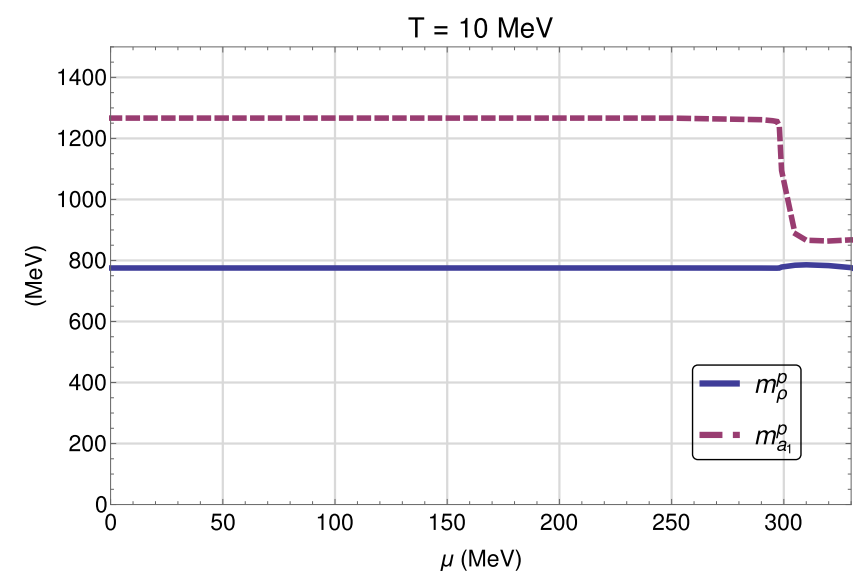

FIG. 14. Chemical potential dependence of the pole masses of $\rho$ (solid blue) and $a_{1}$ (dashed red) mesons at $T=10 \mathrm{MeV}$.

functions at $T=0 \mathrm{MeV}$ and $T=150 \mathrm{MeV}$ are compared to one another in a linear plot. The increasing temperature leads to a decreasing $a_{1}$ pole mass, whereas the peak of the $\rho$ meson stays put as it melts down with temperature. This effect continues by further increasing the temperature until both spectral functions are fully degenerate.

As in the previous study of Ref. [14] this is once again in line with the melting- $\rho$ scenario $[8,12,13,31]$. That the position of the $\rho$-meson resonance is even less temperature dependent here than in the previous study in [14] is seen in Fig. 13 where we plot our estimates of the real parts of the positions of the corresponding poles on the unphysical Riemann sheet as the physical pole masses of $\rho$ and $a_{1}$ mesons over the chiral crossover with increasing temperature.

The corresponding dependence of the same physical pole masses of $\rho$ and $a_{1}$ on the chemical potential at the constant temperature of $T=10 \mathrm{MeV}$ across the critical end point is shown in Fig. 14. The pole mass of the $a_{1}$ also drops considerably in the critical region whereas that of the $\rho$ meson hardly shows much change here either.

\section{SUMMARY AND CONCLUSION}

In this paper we have presented our results from computing in-medium vector and axial-vector meson spectral functions within an effective chirally gauged low-energy theory. Extending the previous study of Ref. [14] we have thereby for the first time also included the fluctuations due to these vector and axial-vector mesons themselves in this nonperturbative computational framework based on the FRG. More specifically, the originally Euclidean FRG flow equations for two-point correlation functions at finite temperature and density are first analytically continued, before they are solved to directly compute retarded Green's functions within this analytically continued aFRG framework.

In order to include the (axial-)vector meson fluctuations in our aFRG flows it was first necessary to understand how off-shell fluctuations due to these massive (axial) vectors are described correctly by elementary fields in an effective theory. Unphysical positivity-violating contributions from longitudinal fluctuations need to be suppressed, and spurious massless single-particle contributions to the transverse fluctuations avoided. Our description is based on the known spectral representations for commutators and causal Green's functions of the corresponding conserved currents, including seagull and Schwinger terms, which are then translated to the (axial-)vector fields of the effective theory via the current-field identities of vector-meson dominance models. This uniquely fixes the form of the transverse single-particle contributions from the massive (axial-)vector fields inside the loops on the right-hand side of the aFRG flow equations. We have implemented these transverse forms, which most importantly do not contain any massless single-particle contributions, together with matching longitudinal components to the (axial-)vector two-point functions which are constructed in a way such that they turn themselves off in the regularized propagators during the flow, as one approaches the limit $k \rightarrow 0$ in the infrared. The procedure of dealing with the longitudinal fluctuations is inspired by the modified Ward identities of FRG flows with current conservation being recovered in the infrared.

Within an effective theory based on the gauged linear sigma model with quarks, we have solved the Euclidean FRG flow for the effective average action in our truncation, as a first step, which is used to determine the thermodynamic potential and the other input needed for the aFRG flows in the second step. For the (axial-)vector fluctuations this input includes scale-dependent mass parameters $m_{v, k}$ and residues $Z_{k} \equiv m_{v, k}^{2} / m_{0, k}^{2}$, i.e., the strengths of the single-particle contributions, in the spirit of an LPA truncation. We have explicitly verified the expected ordering $m_{v, k}^{2} \leq m_{0, k}^{2}$ of these mass parameters together with the 
chiral symmetry breaking pattern in the hierarchy of all Euclidean mass parameters emerging at a typical scale of $k \sim 600 \mathrm{MeV}$ during the flow.

For comparison, we have computed the spectral functions of $\rho$ and $a_{1}$ from the corresponding aFRG flows for the same combinations of temperature and chemical potential as in Ref. [14]. While we observe a similar qualitative overall behavior, with the (axial-)vector fluctuations included, there are now additional processes possible which involve the $\rho$ and $a_{1}$ mesons inside the loops. We have disentangled these various contributions to the spectral functions: For increasing temperature we observe various new capture processes such as $\rho^{*}+\pi \rightarrow a_{1}, a_{1}^{*}+\pi \rightarrow \rho$ and $a_{1}^{*}+\sigma \rightarrow a_{1}$ to dominate the regime between capture and decay processes involving only (pseudo)scalars. The corresponding new decay channels on the other hand produce additional, although subdominant structure in the high frequency domain. As before, at about $T \simeq 300 \mathrm{MeV}$ both spectral functions become completely degenerate.

As a function of chemical potential, the previously observed peak in the critical region, of the $a_{1}$ spectral function at the dropping $a_{1}^{*} \rightarrow \sigma+\pi$ threshold, is still visible on top of all the additional new structure. Perhaps most importantly for further studies, this thus appears to be a robust signature of the critical end point.

In summary, we have developed and tested a successful effective description of (axial-)vector fluctuations due to off-shell single-particle contributions from $\rho$ and $a_{1}$ mesons in agreement with the general spectral properties of the corresponding conserved (axial-)vector currents. From a phenomenological point of view, an important next step will be to implement not only such single-particle contributions in the aFRG flows, but an enhanced ansatz which reflects the full nontrivial spectral properties of a given correlation function, or even a completely self-consistent solution where the nontrivial spectral functions are fed back into the aFRG flows, e.g., via suitable spectral representations. In our present effective theory description, for example, this should lead to the specific peak of the $a_{1}$ spectral function in the critical region to become observable also in the $\rho$-meson spectral function. From there on, this signature can then mix into the electromagnetic spectral function as described in Ref. [40], and hence eventually become an observable feature of thermal dilepton spectra.

\section{ACKNOWLEDGMENTS}

The authors gratefully acknowledge fruitful discussions with Naoto Tanji, Ralf-Arno Tripolt and Jochen Wambach. This work was supported by the Helmholtz International Center (HIC) for FAIR within the LOEWE initiative of the State of Hesse, by the Deutsche Forschungsgemeinschaft (DFG) through Grant No. CRC-TR 211 "Strong-interaction matter under extreme conditions," and by the German Federal Ministry of Education and Research (BMBF), Grant No. 05P18RGFCA.

\section{APPENDIX A: MASSIVE SPIN-1 PARTICLES FROM ANTISYMMETRIC RANK-2 TENSOR FIELDS}

Gasser and Leutwyler have proposed to describe the $\rho$ meson in terms of an antisymmetric rank- 2 tensor field $\rho_{\mu \nu}$ when constructing an effective Lagrangian [45] starting with a free kinetic Lagrangian plus mass term of the form

$$
\mathcal{L}_{0}^{\rho}=-\frac{1}{2}\left(\partial_{\mu} \rho_{\mu \nu}\right) \partial_{\sigma} \rho_{\sigma \nu}+\frac{1}{4} m_{v}^{2} \rho_{\mu \nu} \rho_{\mu \nu}
$$

We are considering just a single flavor component without gauging for simplicity. All we want to point out here is that this Lagrangian, when the components of $\rho_{\mu \nu}$ are reexpressed in terms of a conserved four-vector field, leads to a transverse tree-level two-point function for this vector field in momentum space of the form

$$
\Gamma_{\mu \nu}^{(2) T}(p)=-\frac{m_{v}^{2}}{p^{4}}\left(p^{2}+m_{v}^{2}\right)\left(p^{2} \delta_{\mu \nu}-p_{\mu} p_{\nu}\right),
$$

corresponding to the transverse propagator in Eq. (21) or the corresponding Feynman propagator in Eq. (8).

The essential step to see how this form arises is based on Hodge decomposition and inversion of the de RhamLaplace operator. We therefore sketch it here using the language of de Rham cohomology for brevity. We thus describe classically conserved left- and right-handed $J^{ \pm}$or (axial-)vector $J_{V / A}$ currents in terms of one-forms with

$$
J^{ \pm}=\frac{1}{2}\left(J_{V} \pm J_{A}\right), \quad \text { and } \quad \delta J^{ \pm}=\delta J_{V / A}=0 .
$$

We do not include anomalies in the discussion. By the Poincaré lemma on convex regions of spacetime (we use the Euclidean signature here) these are then coexact, i.e., expressed as exterior coderivatives of (anti-)self-dual twoforms $F^{ \pm}$, with $* F^{ \pm}= \pm F^{ \pm}$,

$$
J^{ \pm}=\delta F^{ \pm}
$$

The self-dual and anti-self-dual antisymmetric rank-2 tensors transform in the $(1,0)$ and $(0,1)$ representations of the Euclidean $O(4)$ [or also the proper orthochronous Lorentz group with $(1,0) \leftrightarrow(0,1)$ under parity, so that $(1,0) \oplus(0,1)$ is used for massive spin-1 particles].

Upon exterior derivation, we thus obtain from Eq. (A4),

$d J^{ \pm}=d \delta F^{ \pm}, \quad$ and $\quad * d J^{ \pm}=* d \delta F^{ \pm}= \pm \delta d F^{ \pm}$.

It is now a simple matter of combining the two and inverting the de Rham-Laplace operator $\Delta=d \delta+\delta d$ to express the field strengths in terms of the conserved currents as 


$$
F^{ \pm}=\Delta^{-1}\left(d J^{ \pm} \pm * d J^{ \pm}\right)
$$

If we want the canonical dimensions of all tensors here to agree with the degree of the corresponding differential form, we identify $F_{\mu \nu}^{+}+F_{\mu \nu}^{-}=m_{\nu} \rho_{\mu \nu}$ in Eq. (A1) to write the corresponding actions for the (anti-)self-dual $F^{ \pm}$of dimension 2 as

$$
\begin{aligned}
S_{0}^{ \pm} & =\int d^{4} x\left\{\frac{1}{4} F_{\mu \nu}^{ \pm} F_{\mu \nu}^{ \pm}-\frac{1}{2 m_{v}^{2}}\left(\partial_{\mu} F_{\mu \nu}^{ \pm}\right) \partial_{\sigma} F_{\sigma \nu}^{ \pm}\right\} \\
& =\frac{1}{4}\left(F^{ \pm}, F^{ \pm}\right)-\frac{1}{2 m_{v}^{2}}\left(\delta F^{ \pm}, \delta F^{ \pm}\right),
\end{aligned}
$$

in the last line also expressed in terms of the Hodge inner product for $p$-forms, e.g.,

$$
(F, F)=\int_{M} F \wedge * F=\int d^{4} x F_{\mu \nu} F_{\mu \nu}
$$

for our two-forms $F$ in $\mathbb{R}^{4}$. The last step now is to likewise rescale our conserved currents, i.e., the coexact one-forms $J^{ \pm}=\delta F^{ \pm}$, which are originally of dimension 3 (they are actually dual to three-forms), with a current-field identity, $J^{ \pm}=m_{v}^{2} V^{ \pm}$, to express them in terms of one-forms $V^{ \pm}$of dimension 1. In terms of these conserved vector fields we then finally obtain from Eq. (A6),

$$
\begin{gathered}
\frac{1}{4}\left(F^{ \pm}, F^{ \pm}\right)=\frac{m_{v}^{4}}{2}\left(V^{ \pm}, \delta \Delta^{-2} d V^{ \pm}\right), \\
\frac{1}{2 m_{v}^{2}}\left(\delta F^{ \pm}, \delta F^{ \pm}\right)=\frac{m_{v}^{2}}{2}\left(V^{ \pm}, \delta \Delta^{-1} d V^{ \pm}\right) .
\end{gathered}
$$

The corresponding two-point functions for the vector fields $V^{ \pm}$are transverse as they must be, and can now be read off from Eq. (A7) with these relations: The mass term from Eq. (A9) produces in momentum space the transverse masslike term in Eq. (A2), i.e., the second one proportional to $m_{v}^{4} / p^{2}$, and the kinetic term from Eq. (A10) produces the first term in Eq. (A2), now actually proportional to $m_{v}^{2}$.

\section{APPENDIX B: FRG FLOW EQUATIONS}

In this Appendix we provide further details on the derivation of the FRG flow equations within our present truncations.

The flow equation for the effective potential $U_{k}\left(\phi^{2}\right)$ is given by

$$
\begin{aligned}
\partial_{k} U_{k}= & \frac{k^{4}}{12 \pi^{2}}\left\{\frac{1+2 n_{B}\left(E_{\sigma, k}\right)}{E_{\sigma, k}}+\frac{3\left(1+2 n_{B}\left(E_{\pi, k}\right)\right)}{E_{\pi, k}}\right. \\
& \left.-\frac{4 N_{\mathrm{f}} N_{\mathrm{c}}}{E_{\psi, k}}\left(1-n_{F}\left(E_{\psi, k}-\mu\right)-n_{F}\left(E_{\psi, k}+\mu\right)\right)\right\},
\end{aligned}
$$

where $n_{B}$ and $n_{F}$ are the bosonic and fermionic occupation numbers, and the scale-dependent quasiparticle energies are defined by

$$
E_{\alpha, k} \equiv \sqrt{k^{2}+m_{\alpha, k}^{2}}, \quad \alpha \in\left\{\pi, \sigma, \rho, a_{1}, \psi\right\} .
$$

The corresponding mass parameters are given by

$$
\begin{gathered}
m_{\pi, k}^{2}=2 U_{k}^{\prime}\left(\phi^{2}\right), \\
m_{\sigma, k}^{2}=2 U_{k}^{\prime}\left(\phi^{2}\right)+4 \phi^{2} U_{k}^{\prime \prime}\left(\phi^{2}\right), \\
m_{\rho, k}^{2}=m_{v, k}^{2}, \\
m_{a_{1}, k}^{2}=m_{v, k}^{2}+g^{2} \phi^{2}, \\
m_{\psi, k}^{2}=h_{s}^{2} \phi^{2},
\end{gathered}
$$

where the derivatives of the scale-dependent effective potential denote derivatives with respect to the chirally invariant $\phi^{2}=\vec{\pi}^{2}+\sigma^{2}$ which are evaluated on the grid in field space when integrating the flow equation for the effective potential.

Evaluated at the scale-dependent minimum $\phi_{0, k}^{2}=\sigma_{0, k}^{2}$ of $U_{k}\left(\phi^{2}\right)$ these mass parameters at the same time yield the scale-dependent Euclidean curvature masses as determined from the respective two-point functions $\Gamma_{\alpha, k}^{(2), E}(p)$ in the limit $p^{2} \rightarrow 0$ which are shown in Fig. 2.

The flow equations for the transverse Euclidean twopoint functions of $\rho$ and $a_{1}$ are given by the projections

$$
\partial_{k} \Gamma_{k}^{(2), T}(p)=\frac{1}{3\left(N_{f}^{2}-1\right)} \Pi_{\mu \nu}^{T}(p) \operatorname{tr}\left(\partial_{k} \Gamma_{\mu \nu, k}^{(2)}(p)\right),
$$

where these equations are evaluated at fixed value in field space, the minimum at the IR scale $\sigma_{0}$. The structure of the flow of $\Gamma_{\rho / a_{1}, k}^{(2)}$ is illustrated in Fig. 1 and contains regulator derivatives $\partial_{k} R_{k}$, vertices $\Gamma_{k}^{(3)}$ and $\Gamma_{k}^{(4)}$ and regulated Euclidean propagators $D_{k}^{E}(q)$.

The regulated propagator of particle species $\alpha$ is defined as

$$
D_{\alpha, k}^{E}(q) \equiv\left(\Gamma_{\alpha, k}^{(2)}(q)+R_{\alpha, k}(q)\right)^{-1},
$$

where we choose three-dimensional analogs of optimized Litim-regulator functions $R_{\alpha, k}(q)$ [66],

$$
\begin{array}{r}
R_{\sigma / \pi, k}(q)=\left(k^{2}-\vec{q}^{2}\right) \Theta\left(k^{2}-\vec{q}^{2}\right), \\
R_{\psi, k}(q)=\mathrm{i} \vec{q}\left(\sqrt{k^{2} / \vec{q}^{2}}-1\right) \Theta\left(k^{2}-\vec{q}^{2}\right), \\
R_{\rho / a_{1}, k}^{T, L}(p)=\frac{-m_{0, k}^{2}}{p^{2}}\left(k^{2}-\vec{p}^{2}\right) \Pi_{\mu \nu}^{T, L}(p) \Theta\left(k^{2}-\vec{q}^{2}\right),
\end{array}
$$


mostly as in previous studies. The vector-meson regulator in Eq. (B12) is chosen in a way so that it acts like a scaleand momentum-dependent mass term which is added to the vector-meson two-point function.

The vertices $\Gamma_{k}^{(3)}$ and $\Gamma_{k}^{(4)}$ are extracted from the ansatz for the effective average action equation (14) and basically contain the couplings $h_{v}, g$ as well as the derivatives of the effective potential. To keep things simple in this qualitative study, the couplings $h_{s}, h_{v}$ and $g$ are chosen to be scale independent as in [14].

We are left with flow equations for the vector-meson masses $m_{v, k}^{2}$ and $m_{0, k}^{2}$. The flow of the mass parameter $m_{0, k}^{2}$ is obtained by projecting the transverse part of the vectormeson two-point function

$$
\Gamma_{\rho, k}^{(2), T}(p)=\frac{-m_{0, k}^{2}}{p^{2}}\left(p^{2}+m_{v, k}^{2}\right),
$$

where the flow equation for $m_{0, k}^{2}$ is obtained from

$$
\begin{aligned}
\partial_{k} m_{0, k}^{2}= & -\frac{1}{3\left(N_{f}^{2}-1\right)} \\
& \times \lim _{p \rightarrow 0} \frac{\partial}{\partial \vec{p}^{2}}\left(p^{2} \Pi_{\mu \nu}^{T}(p) \operatorname{tr}\left(\partial_{k} \Gamma_{\rho, k}^{(2)}(p)\right)_{\mu \nu}\right) .
\end{aligned}
$$

For the flow of $m_{v, k}^{2}$ we first notice that the transverse flow $\partial_{k} \Gamma_{\rho, k}^{(2), T}(p)$ of the vector two-point function is regular for $p \rightarrow 0$ and hence

$$
\begin{aligned}
\partial_{k}\left(m_{v, k}^{2} m_{0, k}^{2}\right)= & -\frac{1}{3\left(N_{f}^{2}-1\right)} \\
& \times \lim _{p \rightarrow 0} p^{2} \Pi_{\mu \nu}^{T}(p) \operatorname{tr}\left(\partial_{k} \Gamma_{\rho, k}^{(2)}(p)\right)_{\mu \nu}=0 .
\end{aligned}
$$

Therefore, one has the simple relation here that

$$
\partial_{k} m_{v, k}^{2}=-\frac{m_{v, k}^{2}}{m_{0, k}^{2}} \partial_{k} m_{0, k}^{2}
$$

These flow equations for $m_{v, k}^{2}$ and $m_{0, k}^{2}$ are usually evaluated at the IR minimum $\sigma_{0}$, i.e., when solving the flow equations for the two-point functions in the spirit of our grid-code techniques. The notable exception are the $k$ dependencies of these masses as shown in Fig. 2 where we have evaluated their flow equations at the $k$-dependent minima $\sigma_{0, k}$ in order to illustrate the scale dependence of the corresponding Euclidean curvature masses.
The flow equations are manipulated and traced using the Mathematica tool FormTracer [67].

\section{APPENDIX C: ANALYTIC CONTINUATION AND SPECTRAL FUNCTIONS}

To obtain flow equations for retarded two-point functions from their Euclidean counterparts we use the same analytic continuation procedure on the level of the flow equations that was developed and also used already for example in $[14,34,37,38]$.

In a first step we exploit the periodicity of the occupation numbers arising in the equations with respect to imaginary and discrete Matsubara modes i $p_{0, n}$,

$$
\begin{aligned}
& n_{B}\left(E \pm \mathrm{i} p_{0, n}\right) \rightarrow n_{B}(E), \\
& n_{F}\left(E \pm \mathrm{i} p_{0, n}\right) \rightarrow n_{F}(E),
\end{aligned}
$$

and then replace this Euclidean energy $p_{0}$ with a real continuous frequency $\omega$

$$
\Gamma^{(2), R}(\omega)=-\lim _{\epsilon \rightarrow 0} \Gamma^{(2), E}\left(p_{0}=-\mathrm{i}(\omega+\mathrm{i} \epsilon)\right) .
$$

For the imaginary part of $\Gamma^{(2), R}$ the limit $\epsilon \rightarrow 0$ can be taken analytically (described in detail in [14]), for the real part of $\Gamma^{(2), R}$ we use $\epsilon=0.1$ or $\epsilon=1$, the $\epsilon$ dependence here is almost negligible. Starting with the following initial values at the UV cutoff scale $\Lambda=1500 \mathrm{MeV}$,

$$
\begin{aligned}
& \Gamma_{\rho, \Lambda}^{(2), R}(\omega)=m_{0, \Lambda}^{2}\left(1-\frac{m_{\rho, \Lambda}^{2}}{(\omega+\mathrm{i} \epsilon)^{2}}\right), \\
& \Gamma_{a_{1}, \Lambda}^{(2), R}(\omega)=m_{0, \Lambda}^{2}\left(1-\frac{m_{a_{1}, \Lambda}^{2}}{(\omega+\mathrm{i} \epsilon)^{2}}\right),
\end{aligned}
$$

the flow equations for $\Gamma_{\rho, k}^{(2), R}$ and $\Gamma_{a_{1}, k}^{(2), R}$ are solved for the real and imaginary parts separately until arriving at the IR scale $k=0 \mathrm{MeV}$. The spectral functions are then given by the imaginary parts of the retarded propagators,

$$
\rho(\omega)=-\frac{1}{\pi} \operatorname{Im} D^{R}(\omega),
$$

which can be expressed in terms of the real and imaginary parts of the retarded two-point functions,

$$
\rho(\omega)=\frac{1}{\pi} \frac{\operatorname{Im} \Gamma^{(2), R}(\omega)}{\left(\operatorname{Re} \Gamma^{(2), R}(\omega)\right)^{2}+\left(\operatorname{Im} \Gamma^{(2), R}(\omega)\right)^{2}} .
$$


[1] P. Braun-Munzinger and J. Wambach, Rev. Mod. Phys. 81, 1031 (2009).

[2] K. Fukushima and T. Hatsuda, Rep. Prog. Phys. 74, 014001 (2011).

[3] E. Shuryak, Rev. Mod. Phys. 89, 035001 (2017).

[4] A. Andronic, P. Braun-Munzinger, K. Redlich, and J. Stachel, Nature (London) 561, 321 (2018).

[5] H. van Hees, J. Weil, S. Endres, and M. Bleicher, EPJ Web Conf. 97, 00028 (2015).

[6] T. Galatyuk, P. M. Hohler, R. Rapp, F. Seck, and J. Stroth, Eur. Phys. J. A 52, 131 (2016).

[7] R. Rapp and H. van Hees, Eur. Phys. J. A 52, 257 (2016).

[8] R. Rapp and J. Wambach, Adv. Nucl. Phys. 25, 1 (2000).

[9] R. Rapp, Proc. Sci., CPOD2013 (2013) 008 [arXiv: 1306.6394].

[10] D. Schildknecht, Acta Phys. Pol. B 37, 595 (2006).

[11] R. Rapp, Adv. High Energy Phys. (London) 2013, 148253 (2013).

[12] R. Rapp, J. Wambach, and H. van Hees, in Relativistic Heavy Ion Physics, edited by R. Stock, Landolt-Börnstein: Numerical Data and Functional Relationships in Science and Technology Vol. 23 (Springer, New York, 2010), p. 134.

[13] P. M. Hohler and R. Rapp, Ann. Phys. (Amsterdam) 368, 70 (2016).

[14] C. Jung, F. Rennecke, R.-A. Tripolt, L. von Smekal, and J. Wambach, Phys. Rev. D 95, 036020 (2017).

[15] H. Gies, Lect. Notes Phys. 852, 287 (2012).

[16] J. Berges, N. Tetradis, and C. Wetterich, Phys. Rep. 363, 223 (2002).

[17] J. M. Pawlowski, Ann. Phys. (Amsterdam) 322, 2831 (2007).

[18] B. Delamotte, Lect. Notes Phys. 852, 49 (2007).

[19] C. Bagnuls and C. Bervillier, Phys. Rep. 348, 91 (2001).

[20] J. M. Pawlowski, AIP Conf. Proc. 1343, 75 (2011).

[21] N. Strodthoff, J. Phys. Conf. Ser. 832, 012040 (2017).

[22] J. Braun, L. Fister, J. M. Pawlowski, and F. Rennecke, Phys. Rev. D 94, 034016 (2016).

[23] A. K. Cyrol, M. Mitter, J. M. Pawlowski, and N. Strodthoff, Phys. Rev. D 97, 054006 (2018).

[24] B.-J. Schaefer and J. Wambach, Nucl. Phys. A757, 479 (2005).

[25] R.-A. Tripolt, B.-J. Schaefer, L. von Smekal, and J. Wambach, Phys. Rev. D 97, 034022 (2018).

[26] B.-J. Schaefer, J. M. Pawlowski, and J. Wambach, Phys. Rev. D 76, 074023 (2007).

[27] K. Fukushima, Phys. Lett. B 591, 277 (2004).

[28] B.-J. Schaefer and M. Mitter, Acta Phys. Pol. B Proc. Suppl. 7, 81 (2014).

[29] F. Rennecke and B.-J. Schaefer, Phys. Rev. D 96, 016009 (2017).

[30] W.-j. Fu, J. M. Pawlowski, and F. Rennecke, arXiv: 1909.02991.

[31] F. Rennecke, Phys. Rev. D 92, 076012 (2015).

[32] J. Eser, M. Grahl, and D. H. Rischke, Phys. Rev. D 92, 096008 (2015).

[33] S. Floerchinger, J. High Energy Phys. 05 (2012) 021.

[34] K. Kamikado, N. Strodthoff, L. von Smekal, and J. Wambach, Eur. Phys. J. C 74, 2806 (2014).

[35] J. M. Pawlowski and N. Strodthoff, Phys. Rev. D 92, 094009 (2015).
[36] R.-A. Tripolt, P. Gubler, M. Ulybyshev, and L. von Smekal, Comput. Phys. Commun. 237, 129 (2019).

[37] R.-A. Tripolt, N. Strodthoff, L. von Smekal, and J. Wambach, Phys. Rev. D 89, 034010 (2014).

[38] R.-A. Tripolt, L. von Smekal, and J. Wambach, Phys. Rev. D 90, 074031 (2014).

[39] J. M. Pawlowski, N. Strodthoff, and N. Wink, Phys. Rev. D 98, 074008 (2018).

[40] R.-A. Tripolt, C. Jung, N. Tanji, L. von Smekal, and J. Wambach, Nucl. Phys. A982, 775 (2019).

[41] R.-A. Tripolt, J. Weyrich, L. von Smekal, and J. Wambach, Phys. Rev. D 98, 094002 (2018).

[42] N. Strodthoff, Phys. Rev. D 95, 076002 (2017).

[43] J. Berges, S. Schlichting, and D. Sexty, Nucl. Phys. B832, 228 (2010).

[44] S. Schlichting, D. Smith, and L. von Smekal, arXiv: 1908.00912.

[45] J. Gasser and H. Leutwyler, Ann. Phys. (N.Y.) 158, 142 (1984).

[46] C. Itzykson and J.-B. Zuber, Quantum Field Theory (McGraw-Hill, New York, 1980).

[47] N. Nakanishi and I. Ojima, Covariant Operator Formalism of Gauge Theories and Quantum Gravity (World Scientific, Singapore, 1990).

[48] J. Frohlich, G. Morchio, and F. Strocchi, Nucl. Phys. B190, 553 (1981).

[49] A. Maas, Prog. Part. Nucl. Phys. 106, 132 (2019).

[50] D. Gross and R. Jackiw, Nucl. Phys. B14, 269 (1969).

[51] T. Watanabe, Prog. Theor. Phys. 47, 2077 (1972).

[52] S. B. Treiman, R. Jackiw, and D. J. Gross, Lectures on Current Algebra and Its Applications (Princeton University Press, Princeton, NJ, 1972).

[53] S. B. Treiman and R. Jackiw, Current Algebra and Anomalies, Princeton Series in Physics Vol. 41 (Princeton University Press, Princeton, NJ, 2016).

[54] J. Schwinger, Phys. Rev. Lett. 3, 296 (1959).

[55] S. Brown, Phys. Rev. 163, 1854 (1967).

[56] D. F. Litim and J. M. Pawlowski, in The Exact Renormalization Group, edited by A. Krasnitz, Y. A. Kubyshin, R. Potting, and P. Sá (World Scientific, Singapore, 1999), p. 168.

[57] J. Weyrich, N. Strodthoff, and L. von Smekal, Phys. Rev. C 92, 015214 (2015).

[58] J. J. Sakurai, Ann. Phys. (N.Y.) 11, 1 (1960).

[59] B. W. Lee and H. T. Nieh, Phys. Rev. 166, 1507 (1968).

[60] M. Urban, M. Buballa, and J. Wambach, Nucl. Phys. A697, 338 (2002).

[61] C. Wetterich, Phys. Lett. B 301, 90 (1993).

[62] N. M. Kroll, T. D. Lee, and B. Zumino, Phys. Rev. 157, 1376 (1967).

[63] Y. Hidaka, O. Morimatsu, and T. Nishikawa, Phys. Rev. D 67, 056004 (2003).

[64] R. A. Tripolt, I. Haritan, J. Wambach, and N. Moiseyev, Phys. Lett. B 774, 411 (2017).

[65] T. D. Cohen, Phys. Rev. Lett. 91, 222001 (2003).

[66] D. F. Litim, Phys. Rev. D 64, 105007 (2001).

[67] A. K. Cyrol, M. Mitter, and N. Strodthoff, Comput. Phys. Commun. 219, 346 (2017). 